\title{
Sensitivity analysis of Wnt $\beta$-catenin based transcription complex might bolster power-logarithmic psychophysical law \& reveal preserved gene gene interactions 站
}

\author{
shriprakash sinha ${ }^{1}$ \\ Faculty of IT and Mathematics \\ Royal Thimphu College, Ngabiphu, P.O. Box-1122, Bhutan
}

\begin{abstract}
Recently, psychophysical laws have been observed to be functional in certain factors working downstream of the Wnt pathway. This work tests the veracity of the prevalence of such laws, albeit at a coarse level, using sensitivity analysis on biologically inspired epigenetically influenced computational causal models. In this work, the variation in the effect of the predictive behaviour of the transcription complex (TRCMPLX) conditional on the evidences of gene expressions in normal/tumor samples is observed by varying the initially assigned values of conditional probability tables (cpt) for TRCMPLX. Preliminary analysis shows that the variation in predictive behaviour of TRCMPLX follows power-logarithmic psychophysical law, crudely. More recently, wet lab experiments have proved the existence of sensors that behave in a logarithmic fashion thus supporting the earlier proposed postulates based on computational sensitivity analysis of this manuscript regarding the existence of logarithmic behaviour in the signaling pathways. It also signifies the importance of systems biology approach where in silico experiments combined with in vivo/in vitro experiments have the power to explore the deeper mechanisms of a signaling pathway. Additionally, it is hypothesized that these laws are prevalent at gene-gene interaction level also. The interactions were obtained by thresholding the inferred conditional probabilities of a gene activation given the status of another gene activation. The deviation in the interactions in normal/tumor samples was similarly observed by varying the initially assigned values of conditional probability tables (cpt) for TRCMPLX. Analysis of deviation in interactions show prevalence of psychophysical laws and

\footnotetext{
${ }^{2}$ Code with dataset is made available under GNU GPL v3 license at google code project on https://sites.google.com/site/shriprakashsinha/shriprakashsinha/ projects/static-bn-for-wnt-signaling-pathway. Please use the scripts in $\mathrm{R}$ as well as the files in zipped directory titled Results-2015.

Email address: ssinha@rtc.bt (shriprakash sinha)

${ }^{1}$ This work was conducted by Sinha as an independent researcher during the period from 2013 to 2016. ORCID : orcid.org/0000-0001-7027-5788
} 
is reported for interaction between elements of pairs (SFRP3, MYC), (SFRP2, CD44) and (DKK1, DACT2). Based on crude static models, it is assumed that dynamic models of Bayesian networks might reveal the phenomena in a better way.

Keywords: Psychophysical laws; Wnt pathway; Causal models; Inference; Bayesian Network; Sensitivity analysis; Gene interaction network

\section{Introduction \& problem statement}

Ever since the accidental discovery of the Wingless in 1973 by Sharma (1973), a tremendous amount of research work has been carried out in the related field of Wnt signaling pathway in the past forty years. A majority of the work has focused on issues related to $\bullet$ the discovery of genetic and epigenetic factors affecting the pathway Thorstensen et al. (2005) \& Baron and Kneissel (2013), • implications of mutations in the pathway and its dominant role on cancer and other diseases Clevers (2006), • investigation into the pathway's contribution towards embryo development Sokol (2011), homeostasis Pinto et al. (2003), Zhong et al. (2014) and apoptosis Pećina-Slaus (2010) and safety and feasibility of drug design for the Wnt pathway Kahn (2014), Garber (2009), Voronkov and Krauss (2012), Blagodatski et al. (2014) \& Curtin and Lorenzi (2010). More recent informative reviews have touched on various issues related to the different types of the Wnt signaling pathway and have stressed not only the activation of the Wnt signaling pathway via the Wnt proteins Rao and Kühl (2010) but also the on the secretion mechanism that plays a major role in the initiation of the Wnt activity as a prelude $\mathrm{Yu}$ and Virshup (2014).

In a more recent development, there has been the observation and study of psychophysical laws prevailing within the pathway and in this regard Goentoro and Kirschner (2009) point to two findings namely, $\bullet$ the robust fold changes of $\beta$-catenin and $\bullet$ the transcriptional machinery of the Wnt pathway depends on the fold changes in $\beta$-catenin instead of absolute levels of the same and some gene transcription networks must respond to fold changes in signals according to the Weber (1834) law in sensory physiology. Note that Weber's law has been found to be a special case of Bernoulli's logarithmic law Masin et al. (2009). If a sensation magnitude $\gamma$ be determined by a stimulus magnitude $\beta$, then the Weber's law states that $\Delta \gamma$ remains constant when the relative stimulus increment $\Delta \beta$ remains constant. The law derives from a more general Bernoullis law were $\Delta \gamma \propto \log \frac{\Delta \beta}{\beta}$. In an unrelated work by Sun et al. (2012), it has been shown that these laws arise at computational level as Bayes optimal under neurobiological constraints at implementational and algorithmic levels. The proposed mathematical framework for understanding the psychophysical scales as Bayes optimal and information theoretically-optimal representation of time sampled continuous valued stimuli is based on established neurobiological assumptions. Sun et al. (2012) also show that the psychophysical laws connect well to quantization frameworks and state that only discrete set of output is distinguishable 
due to biological constraints. This discretization leads to quantization of stimulus also as the nonlinear scaling of the stimulus that leads to the resultant output is invertible. These mathematical insights might explain the indistinguishable insensitive fold changes in levels of $\beta$-catenin shown by Goentoro and Kirschner (2009).

Based on the importance of the revealed phenomena, it might be useful to know if these observations could be verified using computational models apart from analysis of results from wet lab experiments. What is needed is a framework that can capture the causal semantics of the signaling pathway where the influence diagrams involving the interacting extra/intracellular factors working in the pathway, represent the biological knowledge/mechanism of the pathway to a certain extent. Once a model representation is available, the desired variation in the activity of an input factor and the observed variation in the output of the activity of factor(s) can be studied. Sensitivity analysis plays a crucial role in observing the behaviour of output of a variable given variations in the input. As will be seen later, probabilistic graphical models or Bayesian networks provide a framework for representing the causal semantics of the pathway under investigation.

To address these issues, the current work uses the Bayesian network model proposed in Sinha (2014) and conducts sensitivity analysis on the model to check the observations regarding the prevalence of the reported psychophysical laws. In Sinha (2014), it was shown via hypothesis testing that the active (repressed) state of TRCMPLX in the Wnt signaling pathway for colorectal cancer cases is not always correspond to the tumorous (normal) state of the test sample under consideration. For this, Sinha (2014) shows various results on the predicted state of $T R C M P L X$ conditional on the given gene evidences, while varying the assigned probability values of conditional probability tables of TRCMPLX during initialization of the biologically inspired Bayesian Network model. Here, the degree of belief in the activity of TRCMPLX is denoted by the prior probability assigned to the node of $T R C M P L X$ in the network. It was found that the predicted values often increase with an increasing value (in conditional probability tables) of the activity of TRCMPLX on certain genes. What this asks for is that for the recorded deviations due to the changes made in these prior probabilities (i.e the input deviations), is it possible to observe the prevalent logarithmic laws and their deviations (like the Weber's law) as shown by Goentoro and Kirschner (2009), using computational causal modeling?

In this manuscript, the preliminary analysis of deviations computed from variation in prior and estimated conditional probability values using Bayesian network model in Sinha (2014) show that the variation in predictive behaviour of $T R C M P L X$ conditional on gene evidences (i.e the output deviation) follows power and logarithmic psychophysical law crudely, apropos the variation in assigned priors of TRCMPLX (i.e input deviations). This implies that the deviations in output are proportional to increasing function of deviations in input. This relates to the work of Adler et al. (2014) on power and logarithmic law albeit at a coarse level. The granularity is obscured due to the use of static data from Jiang et al. (2008) that is used in Sinha (2014) as well as 
the Bayesian network model that encodes the belief in the factors affecting the pathway in terms of probabilities as well as the inferences made based on the updating of these probabilities conditional on discretized states of gene expression values as evidences. Irrespective of the hurdle posed by the causal models, inferences made based on prior biological knowledge and gene expression evidences coupled with sensitivity analysis sheds light on the prevalent powerlogarithmic psychophysical laws in the pathway. Note that the foundations of the current work were presented as poster in the International Conference on Systems Biology of Human Disease at the German Cancer Research Center in Heidelberg (Germany) in 2015. Followup of some of the implications were shared with a few labs for verification and it is gladdening to see that in a recent development via wet lab experiments by Olsman and Goentoro (2016), it has been confirmed that there are existence of sensors that behave in a logarithmic fashion. The wet lab work by Olsman and Goentoro (2016) supports the earlier proposed crude postulates based on computational sensitivity analysis of this manuscript regarding the existence of logarithmic behaviour in the signaling pathways. It also signifies the importance of systems biology approach where in silico experiments combined with in vivo/in vitro experiments have the power to explore the deeper mechanisms of a signaling pathway.

Adler et al. (2014) show in detail that these laws can be studied empirically using models that exhibit the property of fold change detection (FCD). What this means is that the output depends on the relative changes in the input. The biological feedback models employed for these studies consider various parameters like rates of production of a compound, removal removal of a compound, repression of a compound, levels of scaffolds, kinases, etc. that might be responsible for exhibiting these laws. The current work using the static Bayesian network model might not propose feedback loops directly as used by Adler et al. (2014), yet it could reveal existence of the loops via causal inference even while using static data. The drawback of the current work is its inability to consider cyclic loops. This can be rectified by use of dynamic Bayesian network models that incorporate interaction represented in time series data. Also, the use of Bayesian network models can help in studying the problem from a multiparameter setting as various factors affecting the pathway can be connected in the influence diagrams of the network through the principle of $d$-connectivity/separability. This connectivity will be explained later in the required theory section.

Note that Goentoro and Kirschner (2009) show results for the behaviour of fold change of $\beta$-catenin with respect to changes in the single parameter values i.e the Wnt. On similar lines, the current work takes into account the behaviour of TRCMPLX conditional on affects of multiple parameters in the form of evidences of various intra/extracellular gene expression values working in the pathway, based on the changes made in the assigned prior probabilities for TRCMPLX. The difference here is that one can analyse changes in nodes of a computational model to explore an inherent law in comparison to use of wet lab experiments. The issue here is that FCD which is recorded with respect to changes in levels of concentration can now be recorded via changes in 


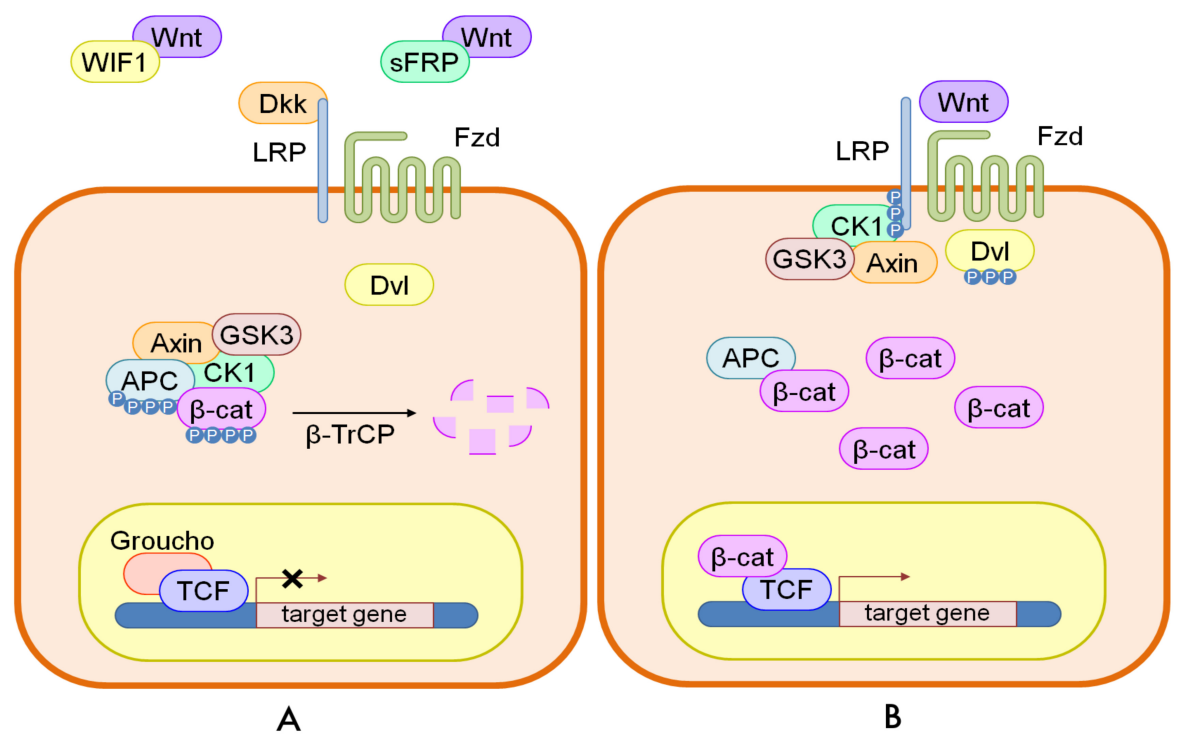

Figure 1: A cartoon of Wnt signaling pathway adapted from Sinha (2014). Part (A) represents the destruction of $\beta$-catenin leading to the inactivation of the Wnt target gene. Part (B) represents activation of Wnt target gene.

the strength of belief in the occurrence of an event. For example, suppose it is not known by what degree the TRCMPLX plays a major role in the signaling pathway quantitatively, then it is possible to encode the degree of belief regarding the role of TRCMPLX in the form of prior or conditional probabilities during initialization of the network. By recording the deviations in these probabilities and observing the output deviations, it is possible to study certain psychophysical laws. Finally, this does not mean that probabilities related to actual concentrations cannot be encoded. Thus, Bayesian networks help in capturing the desired biological knowledge via various causal arcs and conditional probabilities and sensitivity analysis aids in the study of the such natural behaviour.

As a second observation, the forgoing result points towards stability in the behaviour of TRCMPLX and this stability is reflected in the preserved gene gene interactions across the changing values of the priors of TRCMPLX. The interactions are inferred from conditional probabilities of individual gene activation given the status of another gene activation. Finally, as a third observation, it would be interesting to note if the psychophysical laws are prevalent among the dual gene-gene interactions or not. If the results are affirmative then the following important speculations might hold true - Not just one factor but components of the entire network might be exhibiting such a behavior at some stage or the other. • The psychophysical law might not be restricted to individual intra/extracellular components but also to the interactions among the the 
intra/extracellular components in the pathway. This might mean that the interactions manifest during the prevalence of power and logarithmic laws. Further wet lab analysis is needed to very these computational claims.

It is important to be aware of the fact that the presented results are derived from a static Bayesian network model. It is speculated that dynamic models might give much better and more realistic results.

\section{Revisiting the requisite theory}

To understand the logical flow of the current paper, some details of the above related topics from Sinha (2014) are revisited here in order and subdivided into descriptions of - (1) general working of canonical Wnt signaling pathway and some of the involved epigenetic factors (2) introduction to Bayesian networks and (3) the intuition behind the Bayesian network model employed. This is followed by Weber's law and its derivation and finally the notations and terminologies to understand the results and discussion section.

\subsection{Canonical Wnt signaling pathway}

The canonical Wnt signaling pathway is a transduction mechanism that contributes to embryo development and controls homeostatic self renewal in several tissues Clevers (2006). Somatic mutations in the pathway are known to be associated with cancer in different parts of the human body. Prominent among them is the colorectal cancer case Gregorieff and Clevers (2005). In a succinct overview, the Wnt signaling pathway works when the Wnt ligand gets attached to the Frizzled $(f z d) / L R P$ coreceptor complex. $F z d$ may interact with the Dishevelled $(D v l)$ causing phosphorylation. It is also thought that Wnts cause phosphorylation of the $L R P$ via casein kinase $1(C K 1)$ and kinase $G S K 3$. These developments further lead to attraction of Axin which causes inhibition of the formation of the degradation complex. The degradation complex constitutes of Axin, the $\beta$-catenin transportation complex APC, CK1 and GSK3. When the pathway is active the dissolution of the degradation complex leads to stabilization in the concentration of $\beta$-catenin in the cytoplasm. As $\beta$-catenin enters into the nucleus it displaces the Groucho and binds with transcription cell factor $T C F$ thus instigating transcription of Wnt target genes. Groucho acts as lock on $T C F$ and prevents the transcription of target genes which may induce cancer. In cases when the Wnt ligands are not captured by the coreceptor at the cell membrane, Axin helps in formation of the degradation complex. The degradation complex phosphorylates $\beta$-catenin which is then recognized by Fbox $/ W D$ repeat protein $\beta-\operatorname{Tr} C P$. $\beta-\operatorname{Tr} C P$ is a component of ubiquitin ligase complex that helps in ubiquitination of $\beta$-catenin thus marking it for degradation via the proteasome. Cartoons depicting the phenomena of Wnt being inactive and active are shown in figures 1(A) and 1(B), respectively. 


\subsection{Epigenetic factors}

One of the widely studied epigenetic factors is methylation Costello and Plass (2001), Das and Singal (2004), Issa (2007). Its occurrence leads to decrease in the gene expression which affects the working of Wnt signaling pathways. Such characteristic trends of gene silencing like that of secreted frizzled-related proteins $(S F R P)$ family in nearly all human colorectal tumor samples have been found at extracellular level Suzuki et al. (2004). Similarly, methylation of genes in the Dickkopf (DKKx Niehrs (2006), Sato et al. (2007), Dapper antagonist of catenin (DACTx Jiang et al. (2008) and Wnt inhibitory factor-1 (WIF1 Taniguchi et al. (2005) family are known to have significant effect on the Wnt pathway. Also, histone modifications (a class of proteins that help in the formation of chromatin which packs the DNA in a special form Strahl and Allis (2000) can affect gene expression Peterson et al. (2004). In the context of the Wnt signaling pathway it has been found that $D A C T$ gene family show a peculiar behavior in colorectal cancer Jiang et al. (2008). DACT1 and DACT2 showed repression in tumor samples due to increased methylation while $D A C T 3$ did not show obvious changes to the interventions. It is indicated that $D A C T 3$ promoter is simultaneously modified by the both repressive and activating (bivalent) histone modifications Jiang et al. (2008).

\subsection{Bayesian Networks}

In reverse engineering methods for control networks Gardner and Faith (2005) there exist many methods that help in the construction of the networks from the data sets as well as give the ability to infer causal relations between components of the system. A widely known architecture among these methods is the Bayesian Network (BN). These networks can be used for causal reasoning or diagnostic reasoning or both. It has been shown through reasoning and examples in Roehrig (1996) that the probabilistic inference mechanism applied via Bayesian networks are analogous to the structural equation modeling in path analysis problems.

Initial works on BNs in Pearl (1988) and Pearl (2000) suggest that the networks only need a relatively small amount of marginal probabilities for nodes that have no incoming arcs and a set of conditional probabilities for each node having one or more incoming arcs. The nodes form the driving components of a network and the arcs define the interactive influences that drive a particular process. Under these assumptions of influences the joint probability distribution of the whole network or a part of it can be obtained via a special factorization that uses the concept of direct influence and through dependence rules that define d-connectivity/separability as mentioned in Charniak (1991) and Needham et al. (2007). This is illustrated through a simple example in Roehrig (1996).

The Bayesian networks work by estimating the posterior probability of the model given the data set. This estimation is usually referred to as the Bayesian score of the model conditioned on the data set. Mathematically, let $\mathcal{S}$ represent the model given the data $\mathcal{D}$ and $\xi$ is the background knowledge. Then according to the Bayes Theorem Bayes and Price (1763): 


$$
\begin{aligned}
\mathcal{P}(\mathcal{S} \mid \mathcal{D}, \xi) & =\frac{\mathcal{P}(\mathcal{S} \cap \mathcal{D} \mid \xi)}{\mathcal{P}(\mathcal{D} \mid \xi)} \\
& =\frac{\mathcal{P}(\mathcal{S} \mid \xi) \times \mathcal{P}(\mathcal{D} \mid \mathcal{S}, \xi)}{\mathcal{P}(\mathcal{D} \mid \xi)} \\
\text { posterior } & =\frac{\text { prior } \times \text { likelihood }}{\text { constant }}
\end{aligned}
$$

Thus the Bayesian score is computed by evaluating the posterior distribution $\mathcal{P}(\mathcal{S} \mid \mathcal{D}, \xi)$ which is proportional to the prior distribution of the model $\mathcal{P}(\mathcal{S} \mid \xi)$ and the likelihood of the data given the model $\mathcal{P}(\mathcal{D} \mid \mathcal{S}, \xi)$. It must be noted that the background knowledge is assumed to be independent of the data. Next, since the evaluation of probabilities require multiplications a simpler way is to take logarithmic scores which boils down to addition. Thus the estimation takes the form:

$$
\begin{aligned}
\log \mathcal{P}(\mathcal{S} \mid \mathcal{D}, \xi) & =\log \mathcal{P}(\mathcal{S} \mid \xi)+\log \mathcal{P}(\mathcal{D} \mid \mathcal{S}, \xi)-\log \mathcal{P}(\mathcal{D} \mid \xi) \\
& =\log \mathcal{P}(\mathcal{S} \mid \xi)+\log \mathcal{P}(\mathcal{D} \mid \mathcal{S}, \xi)+\text { constant }
\end{aligned}
$$

Finally, the likelihood of the function can be evaluated by averaging over all possible local conditional distributions parameterized by $\theta_{i}$ 's that depict the conditioning of parents. This is equated via:

$$
\begin{aligned}
\mathcal{P}(\mathcal{D} \mid \mathcal{S}, \xi) & =\int_{\theta_{1}} \ldots \int_{\theta_{n}} \mathcal{P}\left(\mathcal{D}, \theta_{i} \mid \mathcal{S}\right) d \theta_{i} \\
& =\int_{\theta_{1}} \ldots \int_{\theta_{n}} \mathcal{P}\left(\mathcal{D} \mid \theta_{i} \mathcal{S}\right) \mathcal{P}\left(\theta_{i} \mid \mathcal{S}\right) d \theta_{i}
\end{aligned}
$$

Work on biological systems that make use of Bayesian networks can also be found in Friedman et al. (2000), Hartemink et al. (2001), Sachs et al. (2002), Sachs et al. (2005) and Peer et al. (2001). Bayesian networks are good in generating network structures and testing a targeted hypothesis which confine the experimenter to derive causal inferences Brent and Lok (2005). But a major disadvantage of the Bayesian networks is that they rely heavily on the conditional probability distributions which require good sampling of datasets and are computationally intensive. On the other hand, these networks are quite robust to the existence of the unobserved variables and accommodates noisy datasets. They also have the ability to combine heterogeneous data sets that incorporate different modalities.

In Sinha (2014), simple static Bayesian Network models have been developed with an aim to show how - incorporation of heterogeneous data can be done to increase prediction accuracy of test samples $\bullet$ prior biological knowledge can be embedded to model biological phenomena behind the Wnt pathway in colorectal cancer $\bullet$ to test the hypothesis regarding direct correspondence of active state 


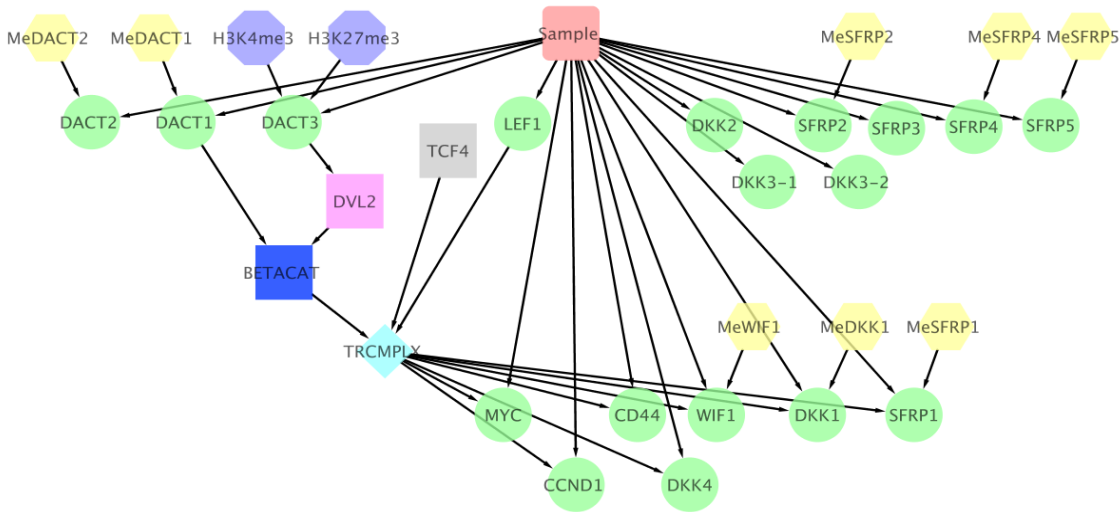

Figure 2: Influence diagram of $\mathcal{M}_{P B K+E I}$ contains partial prior biological knowledge and epigenetic information in the form of methylation and histone modification. Diagram drawn using CYTOSCAPE Shannon et al. (2003). In this model the state of Sample is distinguished from state of TRCMPLX that constitutes the Wnt pathway.

of $\beta$-catenin based transcription complex and the state of the test sample via segregation of nodes in the directed acyclic graphs of the proposed models and - inferences can be made regarding the hidden biological relationships between a particular gene and the $\beta$-catenin transcription complex. This work uses MATLAB implemented BN toolbox from Murphy et al. (2001).

\subsection{Intuition behind the causal semantics of the biologically inspired Bayesian network}

The NB model Ver assumes that the activation (inactivation) of $\beta$-catenin based transcription complex is equivalent to the fact that the sample is cancerous (normal). This assumption needs to be tested and Sinha (2014) proposes a newly improvised models based on prior biological knowledge and epigenetic information regarding the signaling pathway with the assumption that sample prediction may not always mean that the $\beta$-catenin based transcription complex is activated. These assumptions are incorporated by inserting another node of Sample for which gene expression measurements were available. This is separate from the TRCMPLX node that influences a particular set of known genes in the human colorectal cancer. For those genes whose relation with the TRCMPLX is currently not known or biologically affirmed, indirect paths through the Sample node to the TRCMPLX exist, technical aspect of which is described next.

For those factors whose relations were not yet confirmed but known to be involved in the pathway, the causal arcs were segregated via a latent variable introduced into the Bayesian network. The latent variable in the form of Sample (see figure 2) is extremely valuable as it connects the factors whose relations have not been confirmed till now, to factors whose influences have been confirmed 
in the pathway. Finally, the introduction of latent variable in a causal model opens the avenue to assume the presence of measurements that haven't been recorded. Intuitively, for cancer samples the hidden measurements might be different from those for normal samples. The connectivity of factors through the variable provides an important route to infer biological relations.

Since all gene expressions have been measured from a sample of subjects the expression of genes is conditional on the state of the Sample. Here both tumorous and normal cases are present in equal amounts. The transcription factor TRCMPLX under investigation is known to operate with the help of interaction between $\beta$-catenin with TCF4 and LEF1 Waterman (2004), Kriegl et al. (2010). It is also known that the regions in the TSS of $M Y C$ Yochum (2011), CCND1 Schmidt-Ott et al. (2007), CD44 Kanwar et al. (2010), SFRP1 Caldwell et al. (2006), WIF1 Reguart et al. (2004), DKK1 González-Sancho et al. (2004) and $D K K 4$ Pendas-Franco et al. (2008), Baehs et al. (2009) contain factors that have affinity to $\beta$-catenin based TRCMPLX. Thus expression of these genes are shown to be influenced by TRCMPLX, in figure 2 .

Roles of $D K K 2$ Matsui et al. (2009) and $D K K 3$ Zitt et al. (2008), Veeck and Dahl (2012) have been observed in colorectal cancer but their transcriptional relation with $\beta$-catenin based TRCMPLX is not known. Similarly, SFRP2 is known to be a target of Pax 2 transcription factor and yet it affects the $\beta$-catenin Wnt signaling pathway Brophy et al. (2003). Similarly, SF RP4 Feng Han et al. (2006), Huang et al. (2010) and SFRP5 Suzuki et al. (2004) are known to have affect on the Wnt pathway but their role with $T R C M \overline{P L X}$ is not well studied. $S F R P 3$ is known to have a different structure and function with respect to the remaining $S F R P x$ gene family Hoang et al. (1996). Also, the role of DACT2 is found to be conflicting in the Wnt pathway Kivimäe et al. (2011). Thus for all these genes whose expression mostly have an extracellular affect on the pathway and information regarding their influence on $\beta$-catenin based TRCMPLX node is not available, an indirect connection has been made through the Sample node. This connection will be explained at the end of this section.

Lastly, it is known that concentration of DVL2 (a member of Disheveled family) is inversely regulated by the expression of DACT3 Jiang et al. (2008). High $D V L 2$ concentration and suppression of $D A C T 1$ leads to increase in stabilization of $\beta$-catenin which is necessary for the Wnt pathway to be active Jiang et al. (2008). But in a recent development Yuan et al. (2012) it has been found that expression of $D A C T 1$ positively regulates $\beta$-catenin. Both scenarios need to be checked via inspection of the estimated probability values for $\beta$-catenin using the test data. Thus there exists direct causal relations between parent nodes DACT1 and DVL2 and child node, $\beta$-catenin. Influence of methylation (yellow hexagonal) nodes to their respective gene (green circular) nodes represent the affect of methylation on genes. Influence of histone modifications in $H 3 K 27 m e 3$ and $H 3 K 4 m e 3$ (blue octagonal) nodes to DACT3 gene node represents the affect of histone modification on DACT3. The $\beta$-catenin (blue square) node is influenced by concentration of $D V L 2$ (depending on the expression state of $D A C T 3$ ) and behavior of $D A C T 1$.

The aforementioned established prior causal biological knowledge is imposed 


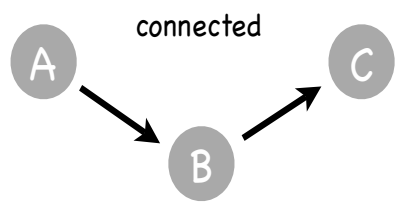

I.
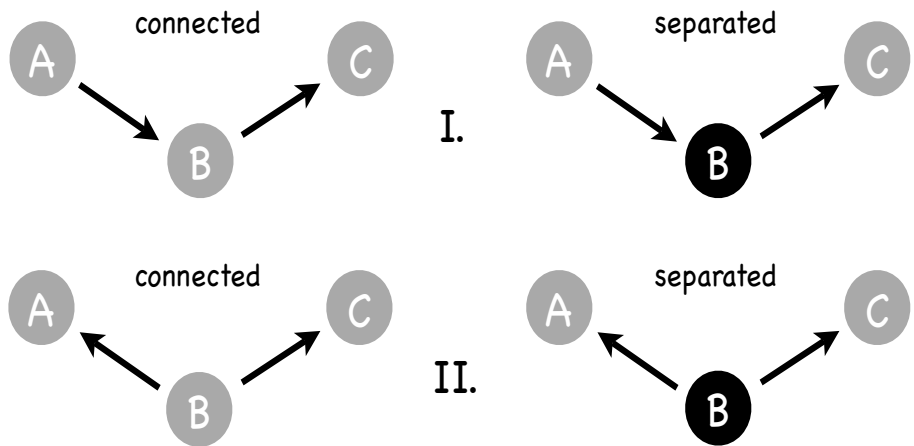

II.
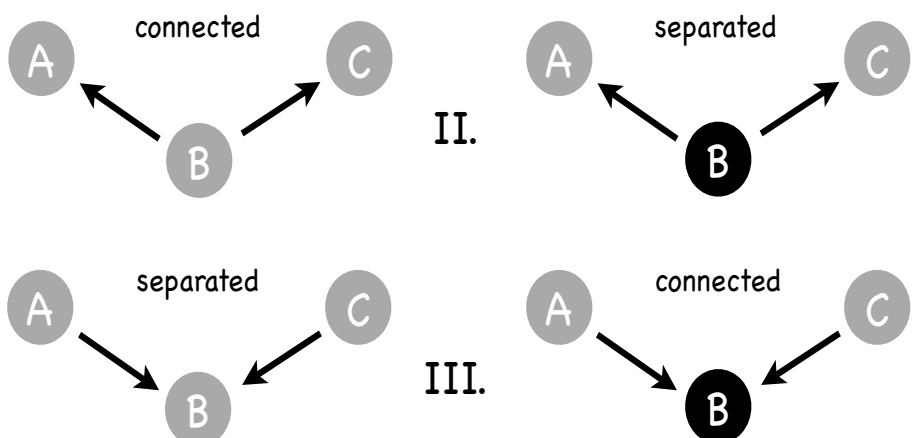

III.

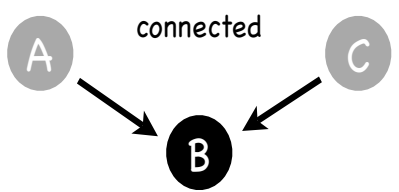

Figure 3: Cases for d-connectivity and d-separation. Black (Gray) circles mean evidence is available (not available) regarding a particular node.

in the Bayesian network model with the aim to computationally reveal unknown biological relationships. The influence diagram of this model is shown in figure 2 with nodes on methylation and histone modification.

In order to understand indirect connections further it is imperative to know about d-connectivity/separability. In a BN model this connection is established via the principle of d-connectivity which states that nodes are connected in a path when there exists no node in the path that has more than one incoming influence edge or there exits nodes in path with more than one incoming influence edge which are observed (i.e evidence regarding such nodes is available) Charniak (1991). Conversely, via principle of $\mathbf{d}$-separation nodes are separated in a path when there exists nodes in the path that have more than one incoming influence edge or there exists nodes in the path with at most one incoming influence edge which are observed (i.e evidence regarding such nodes is available). Figure 3 represents three different cases of connectivity and separation between nodes $\mathcal{A}$ and $\mathcal{C}$ when the path between them passes through node $\mathcal{B}$. Connectivity or dependency exists between nodes $\mathcal{A}$ and $\mathcal{C}$ when $\bullet$ evidence is not present regarding node $\mathcal{B}$ in the left graphs of I. and II. in figure 3 or $\bullet$ evidence is present regarding node $\mathcal{B}$ in the right graph of III. in figure 3. Conversely, separation or independence exits between nodes $\mathcal{A}$ and $\mathcal{C}$ when - evidence is present regarding node $\mathcal{B}$ in the right graphs of I. and II. in figure 3 or $\bullet$ evidence is not present regarding node $\mathcal{B}$ in the left graph of III. in figure 3. 
It would be interesting to know about the behaviour of $T R C M P L X$ given the evidence of state of $S F R P 3$. To reveal such information paths must exist between these nodes. It can be seen that there are multiple paths between TRCMPLX and SFRP2 in the BN model in figure 2. These paths are enumerated as follows:

1. SFRP3, Sample, SFRP1, TRCMPLX

2. SFRP3, Sample, DKK1, TRCMPLX

3. SFRP3, Sample, WIF1, TRCMPLX

4. SFRP3, Sample, CD44, TRCMPLX

5. SFRP3, Sample, DKK4, TRCMPLX

6. SFRP3, Sample, CCND1, TRCMPLX

7. SFRP3, Sample, MYC, TRCMPLX

8. SFRP3, Sample, LEF1, TRCMPLX

9. SFRP3, Sample, DACT3, DVL2, $\beta$-catenin, TRCMPLX

10. SFRP3, Sample, DACT1, $\beta$-catenin, TRCMPLX

Knowledge of evidence regarding nodes of $S F R P 1$ (path 1), DKK1 (path 2), $W I F 1$ (path 3), CD44 (path 4), DKK4 (path 5), CCND1 (path 6) and $M Y C$ (path 7) makes Sample and TRCMPLX dependent or d-connected. Further, no evidence regarding state of Sample on these paths instigates dependency or connectivity between $S F R P 3$ and TRCMPLX. On the contrary, evidence regarding $L E F 1, D A C T 3$ and DACT1 makes Sample (and child nodes influenced by Sample) independent or d-separated from TRCMPLX through paths (8) to (10). Due to the dependency in paths (1) to (7) and the given state of SFRP3 (i.e evidence regarding it being active or passive), the BN uses these paths during inference to find how TRCMPLX might behave in normal and tumorous test cases. Thus, exploiting the properties of d-connectivity/separability, imposing a biological structure via simple yet important prior causal knowledge and incorporating epigenetic information, BN help in inferring many of the unknown relation of a certain gene expression and a transcription complex.

\subsection{The logarithmic psychophysical law}

Masin et al. (2009) states the Weber's law as follows -

Consider a sensation magnitude $\gamma$ determined by a stimulus magnitude $\beta$. Fechner (1860) (vol 2, p. 9) used the symbol $\Delta \gamma$ to denote a just noticeable sensation increment, from $\gamma$ to $\gamma+\Delta \gamma$, and the symbol $\Delta \beta$ to denote the corresponding stimulus increment, from $\beta$ to $\beta+\Delta \beta$. Fechner (1860) (vol 1, p. 65) attributed to the German physiologist Ernst Heinrich Weber the empirical finding Weber (1834) that $\Delta \gamma$ remains constant when the relative stimulus increment $\frac{\Delta \beta}{\beta}$ remains constant, and named this finding Weber's law. Fechner (1860) (vol 2, p. 10) underlined that Weber's law was empirical. 
It has been found that Bernoulli's principle Bernoulli (1738) is different from Weber's law Weber (1834) in that it refers to $\Delta \gamma$ as any possible increment in $\gamma$, while the Weber's law refers only to just noticeable increment in $\gamma$. Masin et al. (2009) shows that Weber's law is a special case of Bernoulli's principle and can be derived as follows - Equation 4 depicts the Bernoulli's principle and increment in sensation represented by $\Delta \gamma$ is proportional to change in stimulus represented by $\Delta \beta$.

$$
\gamma=b \times \log \frac{\beta}{\alpha}
$$

were $b$ is a constant and $\alpha$ is a threshold. To evaluate the increment, the following equation 5 and the ensuing simplification gives -

$$
\Delta \gamma=b \times \log \frac{\beta+\Delta \beta}{\alpha}-b \times \log \frac{\beta}{\alpha}=b \times \log \left(\frac{\beta+\Delta \beta}{\beta}\right)=b \times \log \left(1+\frac{\Delta \beta}{\beta}\right)
$$

Since $b$ is a constant, equation 5 reduces to

$$
\Delta \gamma \circ \frac{\Delta \beta}{\beta}
$$

were o means "is constant when there is constancy of" from Masin et al. (2009). The final equation 6 is a formulation of Weber's laws in wordings and thus Bernoulli's principles imply Weber's law as a special case. Using Fechner (1860) derivation, it is possible to show the relation between Bernoulli's principles and Weber's law. Starting from the last line of equation 5 , the following yields the relation.

$$
\begin{aligned}
\Delta \gamma & =b \times \log \left(1+\frac{\Delta \beta}{\beta}\right) \Longrightarrow e^{\Delta \gamma}=e^{b \times \log \left(1+\frac{\Delta \beta}{\beta}\right)} \\
k_{p} & =e^{\log \left(1+\frac{\Delta \beta}{\beta}\right)^{b}} ; \text { were } k_{p}=e^{\Delta \gamma} \Longrightarrow k_{p}=\left(1+\frac{\Delta \beta}{\beta}\right)^{b} ; \text { since } e^{\log (x)}=x \\
\sqrt[b]{k_{p}} & =1+\frac{\Delta \beta}{\beta} \\
k_{q}-1 & =\frac{\Delta \beta}{\beta} ; \text { were } \sqrt[b]{k_{p}}=k_{q} \Longrightarrow k_{r}=\frac{\Delta \beta}{\beta} ; \text { the weber's law s.t. } k_{r}=\sqrt[b]{e^{\Delta \gamma}}-1
\end{aligned}
$$

Equation 6 holds true given the last line of equation 7 In the current study, observation of deviation recorded in predicted values of state of TRCMPLX conditional on gene evidences show crude logarithmic behaviour which might bolster Weber's law and Bernoulli's principles. But it must be noted that these observations are made on static causal models and observation of the same behaviour in dynamical setting would add more value.

\section{Materials and methods}

The models purported by Sinha (2014) involving the biological knowledge as well as epigenetic information depicted by $\mathcal{M}_{P B K+E I}$ and biological knowl- 
edge excluding epigenetic information $\mathcal{M}_{P B K}$ were used to predict the state of TRCMPLX given the gene evidences. Figure 2 depicts the model $\mathcal{M}_{P B K+E I}$. The predictions were recorded over the varying effect of TRCMPLX on gene regulations via assignment of different values to conditional probability tables (cpt) of TRCMPLX while initializing the aforementioned BN models. This varying effect is represented by the term ETGN in Sinha (2014).

As a recapitulation, the design of the experiment is a simple 2-holdout experiment where one sample from the normal and one sample from the tumorous are paired to form a test dataset. Excluding the pair formed in an iteration of 2-hold out experiment the remaining samples are considered for training of a BN model. Thus in a data set of 24 normal and 24 tumorous cases obtained from Jiang et al. (2008), a training set will contain 46 samples and a test set will contain 2 samples (one of normal and one of tumor). This procedure is repeated for every normal sample which is combined with each of the tumorous sample to form a series of test datasets. In total there will be 576 pairs of test data and 576 instances of training data. Note that for each test sample in a pair, the expression value for a gene is discretized using a threshold computed for that particular gene from the training set. Computation of the threshold has been elucidated in Sinha (2014). This computation is repeated for all genes per test sample. Based on the available evidence from the state of expression of all genes, which constitute the test data, inference regarding the state of both the TRCMPLX and the test sample is made. These inferences reveal information regarding the activation state of the TRCMPLX and the state of the test sample. Finally, for each gene $g_{i}$, the conditional probability $\operatorname{Pr}\left(g_{i}=\right.$ active $\mid g_{k}$ evidence) $\forall k$ genes. Note that these probabilities are recorded for both normal and tumor test samples.

Three observations are presented in this manuscript. The first observation is regarding the logarithmic deviations in prediction of activation status of TRCMPLX conditional on gene expression evidences. The second observation is preservation of some gene gene interactions across different strength of beliefs concerning the affect of TRCMPLX. To observe these preservations, first the gene gene interactions have to be constructed from the predicted conditional probabilities of one gene given the evidence of another gene (for all gene evidences taken separately). After the construction, further preprocessing is required before the gene-gene interaction network can be inferred. Finally, the third observation is to check whether these laws are prevalent among the gene-gene interactions in the network or not.

\section{Results and discussion on observation 1}

\subsection{Logarithmic-power deviations in predictions of $\beta$-catenin transcription com- plex}

Let $\gamma$ be $\operatorname{Pr}(T R C M P L X=$ active|all gene evidences $), \beta$ be the assigned cpt value of TRCMPLX during initialization of the Bayesian network models and $\Delta \beta$ be the deviation in the assigned values of TRCMPLX during initialization. 


\begin{tabular}{|c|c|c|c|c|c|}
\hline \multicolumn{6}{|c|}{ Deviation study for model $\mathcal{M}_{P B K+E I}$} \\
\hline$\beta$ & $\Delta \beta$ & $\frac{\Delta \beta}{\beta}$ & $\log \left(1+\frac{\Delta \beta}{\beta}\right)$ & $\Delta \gamma$ in Normal & $\Delta \gamma$ in Tumor \\
\hline 0.8 & 0.1 & 0.125 & 0.117783 & 0.03055427 & 0.09151151 \\
\hline 0.7 & 0.1 & .1428571 & 0.1335314 & 0.01423754 & 0.09086427 \\
\hline 0.6 & 0.1 & .1666667 & 0.1541507 & 0.004384244 & 0.08052346 \\
\hline 0.5 & 0.1 & 0.2 & 0.1823216 & 0.0005872203 & 0.07294716 \\
\hline 0.8 & 0.1 & 0.125 & 0.117783 & 0.03055427 & 0.09151151 \\
\hline 0.7 & 0.2 & .2857143 & 0.2513144 & 0.04479181 & 0.1823758 \\
\hline 0.6 & 0.3 & 0.5 & 0.4054651 & 0.04917605 & 0.2628992 \\
\hline 0.5 & 0.4 & 0.8 & 0.5877867 & 0.04976327 & 0.3358464 \\
\hline
\end{tabular}

Table 1: Deviation study for model $\mathcal{M}_{P B K+E I} . \Delta \gamma-$ mean value of $\operatorname{Pr}(T R C M P L X=$ active $\mid \forall g e_{i}$ evidences) over all runs, $\gamma-\operatorname{Pr}(T R C M P L X=$ active $\mid$ all gene evidences $), \beta$ - the assigned cpt value of TRCMPLX during initialization of the Bayesian network models and $\Delta \beta$ - the deviation in the assigned values of TRCMPLX during initialization.

To compute $\Delta \gamma$, the 576 predictions of $\gamma$ observed at $\beta=90 \%$ is subtracted from the 576 predictions of $\gamma$ observed at $\beta=80 \%$ and a mean of the deviations recorded. This mean becomes $\Delta \gamma$. The procedure is computed again for different value of $\beta$. In this manuscript, the effect of constant and incremental deviations are observed. Tables 11 and 2 represent the deviations for models $\mathcal{M}_{P B K+E I}$ and $\mathcal{M}_{P B K}$, respectively.

Figures 4, 5, 6 and 7 show the deviations represented in tables 1 and 2, Note that the numbers depicted in the tables are scaled in a nonuniform manner for observational purpose in the figures. Unscaled values are represented under the last two columns on the right of tables 1 and 2 . Before reading the graphs, note that red indicates deviation of mean of $\operatorname{Pr}\left(T R C M P L X=\right.$ active $\forall g e_{i}$ evidences $)$ in normal test samples, blue indicates deviation of mean of $\operatorname{Pr}(T R C M P L X=$ active $\mid \forall g e_{i}$ evidences) in tumor case, green indicates deviations in Weber's law and cyan indicates deviations in Bernoulli's law.

For the case of contant deviations (figure 44 in model $\mathcal{M}_{P B K+E I}$, it was observed that deviations in activation of TRCM PLX conditional on gene evidences for the tumor test samples showed a logarithmic behaviour and were directly proportional to the negative of both the Weber's and Bernoulli's law. This can be seen by the blue curve almost following the green and cyan curves. For the case of deviations in activation of TRCMPLX conditional on gene evidences for the normal test samples showed an exponential behaviour and were proportional to negative of both the Weber's and Bernoulli's law. Similar behaviour was observed for all the coloured curves in case of incremental deviations as shown in figure 5 . The exponential behaviour for activation of TRCMPLX being active conditional on gene evidences correctly supports to the last line of 


\begin{tabular}{|c|c|c|c|c|c|}
\hline$\beta$ & $\Delta \beta$ & $\frac{\Delta \beta}{\beta}$ & $\log \left(1+\frac{\Delta \beta}{\beta}\right.$ & $\Delta \gamma$ in Norma & $\Delta \gamma$ in Tumor \\
\hline$\overline{0.8}$ & 0.1 & 0.125 & 0.117783 & 0.1400355 & 0.1097089 \\
\hline 0.7 & 0.16 & 0.1428571 & 0.1335314 & 0.06442086 & 0.1877266 \\
\hline 0.6 & $0.1 \mathrm{C}$ & 0.1666667 & 0.1541507 & 0.01762791 & 0.06204044 \\
\hline 0.5 & 0.1 & 0.2 & 0.1823216 & 0.01393517 & 0.1718198 \\
\hline 0.8 & 0.1 & 0.125 & 0.117783 & 0.1400355 & 0.1097089 \\
\hline 0.7 & 0.2 & 0.2857143 & 0.2513144 & 0.2044564 & 0.2974356 \\
\hline 0.6 & 0.3 & 0.5 & 0.4054651 & 0.2220843 & 0.359476 \\
\hline 0.5 & 0.4 & 0.8 & 0.5877867 & 0.2360195 & 0.5312958 \\
\hline
\end{tabular}

Table 2: Deviation study for model $\mathcal{M}_{P B K} . \Delta \gamma$ - mean value of $\operatorname{Pr}\left(T R C M P L X=\right.$ active $\mid \forall g e_{i}$ evidences) over all runs, $\gamma-\operatorname{Pr}(T R C M P L X=$ active|all gene evidences $), \beta$ - the assigned cpt value of TRCMPLX during initialization of the Bayesian network models and $\Delta \beta$ - the deviation in the assigned values of TRCMPLX during initialization.

equation 7 which is the derivation of Weber's law from Bernoulli's equation. It actually point to Fechner's derivation of Weber's law from logarithmic formulation.

For model $\mathcal{M}_{P B K}$, the above observations do not yield consistent behaviour. In figure 6, for the case of constant deviations, only the deviations in activation of TRCMPLX conditional on gene evidences for normal test samples exponential in nature and were found to be directly proportional to the negative of both the Weber's and Bernoulli's law. But the deviations in activation of TRCMPLX conditional on gene evidences in tumor test samples show noisy behaviour. But this observation is not the case in incremental deviations for the same model. For the case of incremental deviations as represented in figure 7. the deviations in activation of TRCMPLX conditional on gene evidences is directly proportional to both the Weber's and Bernoulli's law. The figure actually represent the plots with inverted values i.e negative values. A primary reason for this behaviour might be that $\mathcal{M}_{P B K}$ does not capture and constrain the network as much as $\mathcal{M}_{P B K+E I}$ which include epigenetic information. This inclusion of heterogeneous information adds more value to the biologically inspired network and reveals the hidden natural laws occurring in the signaling pathway in both normal and tumor cases.

\subsection{Intuition behind the curve behaviour}

Lastly, the intuitive idea behind the behaviour of the curves generated from constant deviation in table 1 is as follows. It is expected that $\operatorname{Pr}(T R C M P L X=$ active/all gene evidences) is low (high) in the case of Normal (Tumor) samples. The change $\triangle \operatorname{Pr}(T R C M P L X=$ active|all gene evidences $)$ jumps by power of 


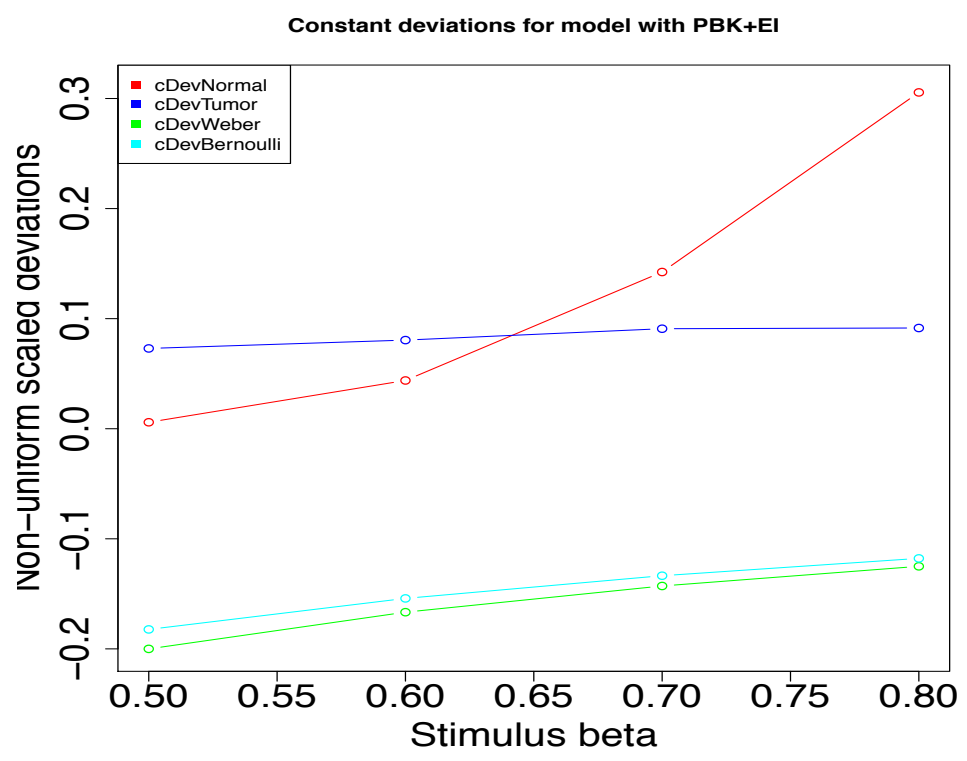

Figure 4: Constant deviations in $\beta$ i.e ETGN and corresponding deviations in $\operatorname{Pr}(T R C M P L X$ $=$ active $\mid \forall g e_{i}$ evidences) for both normal and tumor test samples. Corresponding Weber and Bernoulli deviations are also recorded. Note that the plots and the y-axis depict scaled deviations to visually analyse the observations. The model used is $\mathcal{M}_{P B K+E I}$. Red - constant deviation in Normal, constant deviation in Tumor, Green - constant deviation in Weber's law, Cyan - constant deviation in Bernoulli's law.

10 as the $\beta$ values change from $50 \%$ to $90 \%$ in Normal cases. It can be observed from the table that there are low deviations in $\operatorname{Pr}(T R C M P L X=$ active|all gene evidences) when $\beta$ is low i.e the effect of transcription complex is low and high deviations in $\operatorname{Pr}(T R C M P L X=$ active|all gene evidences) when $\beta$ is high i.e the effect of transcription complex is high. But it should be noted that the deviations still tend to be small. This implies that the TRCMPLX is switched off at a constant rate. Thus changes in $\beta$ leads to exponential curves as in the formulation $\frac{\Delta \beta}{\beta}, \Delta \beta \rightarrow 0$ and $\beta \rightarrow \infty$.

In tumor cases, $\triangle \operatorname{Pr}(T R C M P L X=$ active|all gene evidences $)$ behaves near to logarithmic curve as $\beta$ increases from $50 \%$ to $90 \%$. The deviations increase in a slow monotonic way as $\beta$ increases. Finally, the ratio $\frac{\Delta \beta}{\beta}$ shows monotonically increasing behaviour as $\Delta \beta$ increases proportionally with $\beta$. This means that in tumor samples the rate of transcription increases or the effect of rate of transcription complex increases monotonically as $\beta$ increases. This points to the fold change in $\beta$-catenin concentration that might be influencing the transcription rate of the transcription complex. In normal case, the $\beta$-catenin concentration remains constant. Due to this, changes in the rate of transcription by the transcription complex might remains constant and near to zero. Change in $\beta$ values that is the change in initialization of cpt values of transcription complex causes 


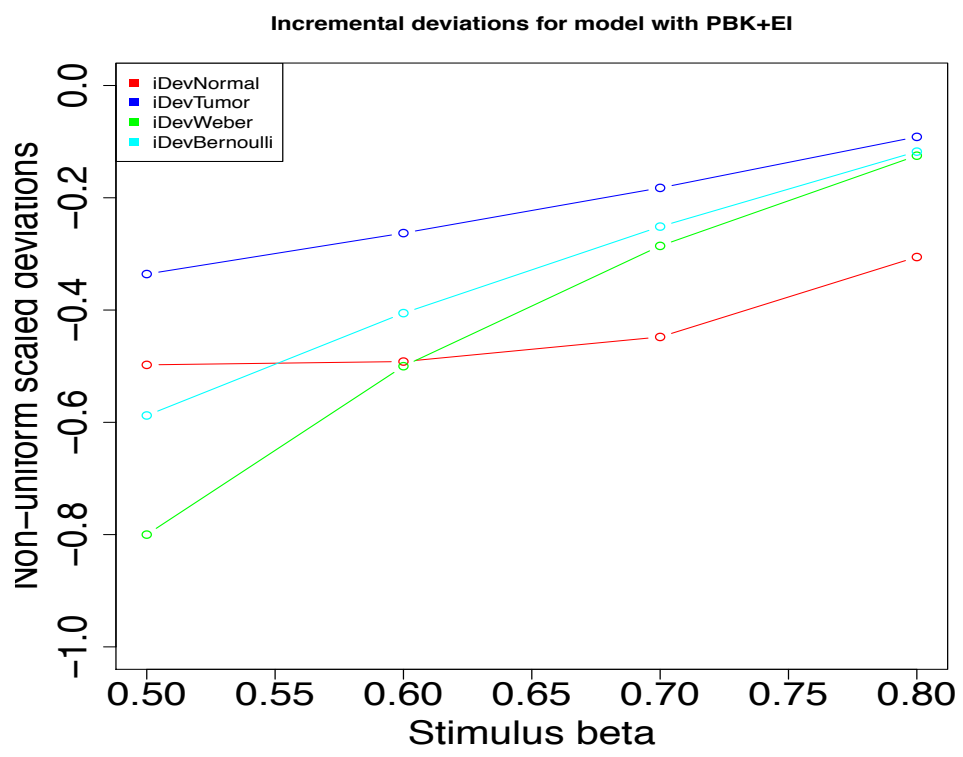

Figure 5: Incremental deviations in $\beta$ i.e ETGN and corresponding deviations in $\operatorname{Pr}\left(T R C M P L X=\right.$ active $\mid \forall g e_{i}$ evidences) for both normal and tumor test samples. Corresponding Weber and Bernoulli deviations are also recorded. Note that the plots and the y-axis depict scaled deviations to visually analyse the observations. The model used is $\mathcal{M}_{P B K+E I}$. Red - incremental deviation in Normal, incremental deviation in Tumor, Green - incremental deviation in Weber's law, Cyan - incremental deviation in Bernoulli's law.

the logarithmic curve in deviations of prediction of transcription complex.

Finally, these observations present a crude yet important picture regarding the downstream transcriptional behaviour of signaling pathway in case of colorectal cancer. Though the current model does not measure the fold changes in the concentration levels of $\beta$-catenin, it can help in measuring the deviations in activity of the transcription complex conditional on the gene evidences by observing the deviations in the strength of belief assigned as priors in the probability tables of the node representing the transcription complex of the network. Thus sensitivity analysis facilitates in observing such natural phenomena at computational level. In context of the work by Goentoro and Kirschner (2009), the presented results are crude in terms of static observations yet they show corresponding behaviour of transcriptional activity in terms of psychophysical laws. Further investigations using dynamic models might reveal more information in comparison to the static models used in Sinha (2014). The observations presented here bolster the existence of behavioural phenomena in terms of logarithmic laws. 


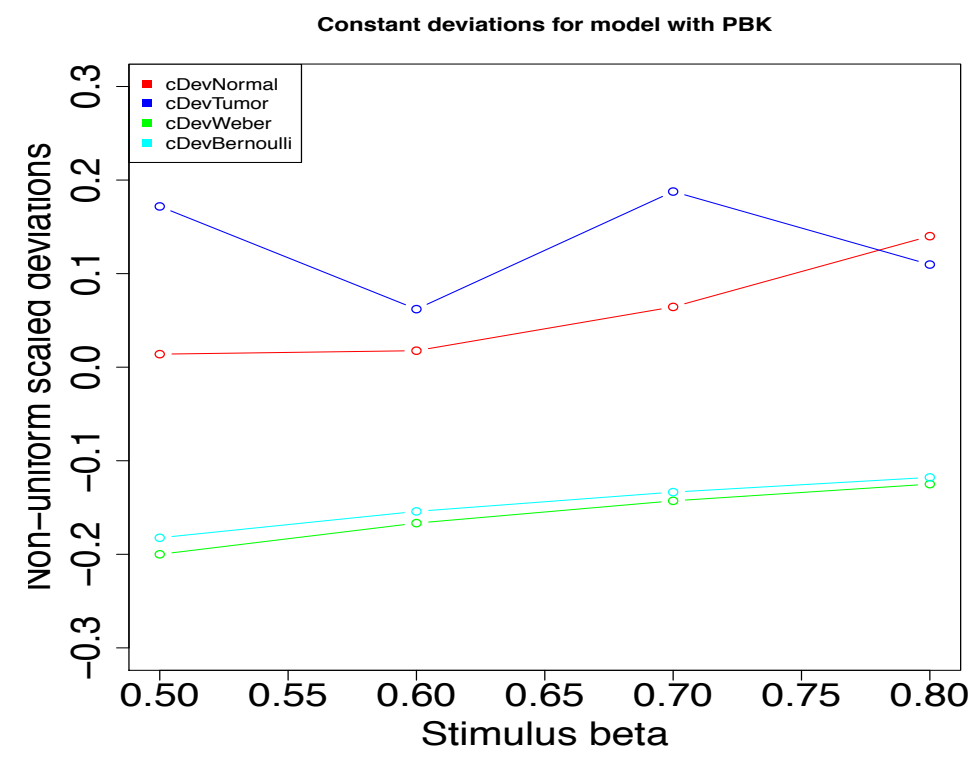

Figure 6: Constant deviations in $\beta$ i.e ETGN and corresponding deviations in $\operatorname{Pr}(T R C M P L X$ $=$ active $\mid \forall g e_{i}$ evidences) for both normal and tumor test samples. Corresponding Weber and Bernoulli deviations are also recorded. Note that the plots and the y-axis depict scaled deviations to visually analyse the observations. The model used is $\mathcal{M}_{P B K}$. Red - constant deviation in Normal, constant deviation in Tumor, Green - constant deviation in Weber's law, Cyan - constant deviation in Bernoulli's law.

\section{Preservation of gene gene interactions}

The second part of this study was to find interactions between two genes by observing the conditional probability of activation status of one gene given the evidence of another gene. Let $g$ be a gene. To obtain the results, two steps need to be executed in a serial manner. The first step is to construct gene gene interactions based on the available conditional probabilities denoted by $\operatorname{Pr}\left(g_{i}=\right.$ active/repressed $\mid g_{k}$ evidence) $\forall k$ genes. The conditional probabilities are inferred using the junction tree algorithm that employs two-pass message passing scheme. Example code and implementations of the same can be found in Murphy et al. (2001). The steps for constructing the gene gene interactions based on these conditional probabilities are documented in the Appendix. The second step is to infer gene gene interaction network based purely on reversible interactions. Note that networks are inferred for gene evidences using normal and tumor test samples separately.

Finally, once the interaction network is ready, the computational empirical estimates for deviations in gene-gene interaction is recorded and observation on the prevalence of psychophysical laws in these interactions is discussed. An important point that needs to be kept in mind is that the inferred interaction network differs based on the choice of the threshold involved (which is a 


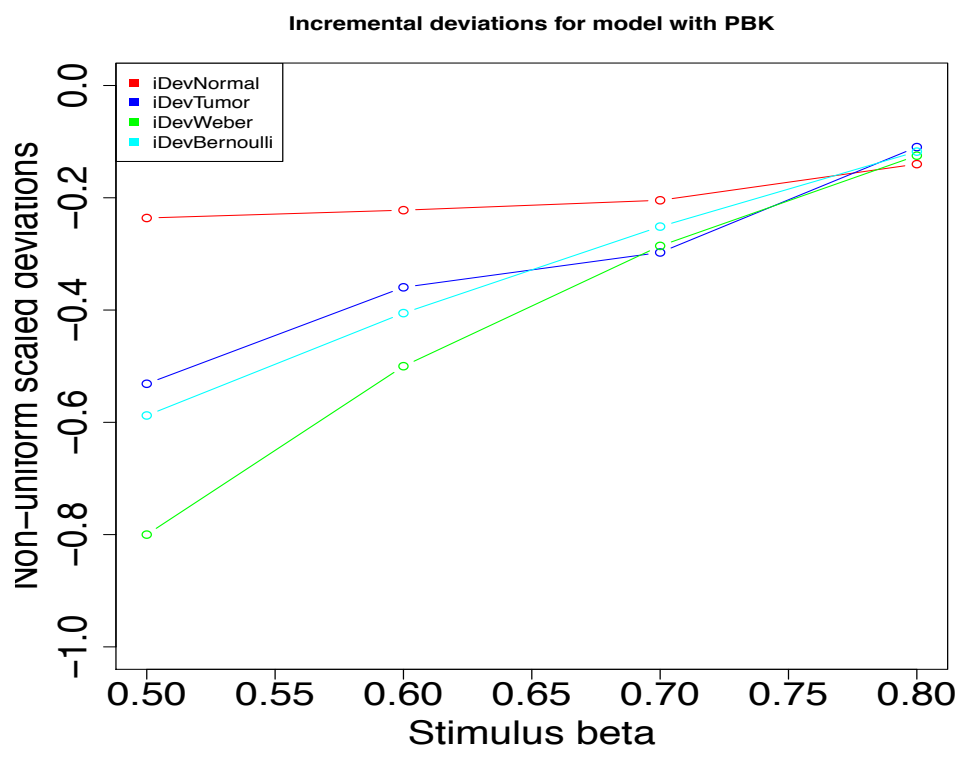

Figure 7: Incremental deviations in $\beta$ i.e ETGN and corresponding deviations in $\operatorname{Pr}\left(T R C M P L X=\right.$ active $\mid \forall g e_{i}$ evidences) for both normal and tumor test samples. Corresponding Weber and Bernoulli deviations are also recorded. Note that the plots and the $\mathrm{y}$-axis depict scaled deviations to visually analyse the observations. The model used is $\mathcal{M}_{P B K}$. Red - incremental deviation in Normal, incremental deviation in Tumor, Green - incremental deviation in Weber's law, Cyan - incremental deviation in Bernoulli's law.

computational issue) but the underlying psychophysical laws remain unchanged (which is a natural phenomena irrespective of the components involved). Thus the while reading the observations on the psychophysical laws, readers must not get confused regarding plots made for interactions from different networks.

\subsection{Constructing gene-gene interactions}

Gene interactions are constructed by labeling the inferred conditional probability of activation of $g_{j}$ given the state of $g_{i}$, for all $j^{\prime} s \& i^{\prime} s$. Here labels refer to assigning $<>$ for an activated gene and $\mid$ for repressed gene. Thus the following possible combinations can be inferred $-g_{j}<>-<>g_{i}, g_{j}|-| g_{i}, g_{j}<>-\mid g_{i}$ and $g_{j} \mid-<>g_{i}$. Note that all interactions are basically depicting the degree of belief in the state of $g_{i}$ given or conditional on $g_{j}$ i.e $\operatorname{Pr}\left(g_{i} \mid g_{j}\right)$. The label related to $g_{i}$ is derived by discretizing $\operatorname{Pr}\left(g_{i} \mid g_{j}\right)$ with respect to a weighted mean or the arbitrary value of 0.5 . In any interaction, the label associated with $g_{j}$ is the evidence and the label associated with $g_{i}$ is the predicted conditional probability. Thus there will always exist two way interactions corresponding to $\operatorname{Pr}\left(g_{j} \mid g_{i}\right)$ and $\operatorname{Pr}\left(g_{i} \mid g_{j}\right)$ in a Normal case. Similar interactions can be inferred for the Tumor case. Which interactions to select is based on criteria of reversibility and duplication, which is addressed later. To reiterate a final note regarding the 


\begin{tabular}{|c|c|c|c|c|c|c|c|c|c|c|c|}
\hline \multicolumn{12}{|c|}{ SFRP 3 activation status apropos gene evidences in Normal and Tumor samples using $\theta=0.5$} \\
\hline & ge & $a a_{N}$ & $a r_{N}$ & $r a_{N}$ & $r r_{N}$ & $a a_{T}$ & $a r_{T}$ & $r a_{T}$ & $r r_{T}$ & $g g_{I N}$ & $g g_{I T}$ \\
\hline 1 & DKK1 & 192 & 360 & 24 & 0 & 0 & 216 & 360 & 0 & DKK1 $\mid-<>$ SFRP3 & DKK1 $<>-\mid$ SFRP3 \\
\hline 2 & DKK2 & 360 & 168 & 0 & 48 & 216 & 217 & 0 & 143 & DKK2 $<>-<>$ SFRP3 & DKK2 $\mid-<>$ SFRP3 \\
\hline 3 & DKK3-1 & 360 & 160 & 0 & 56 & 216 & 226 & 0 & 134 & DKK3-1 $<>-<>$ SFRP3 & DKK3-1 $\mid-<>$ SFRP3 \\
\hline 4 & DKK3-2 & 240 & 336 & 0 & 0 & 336 & 240 & 0 & 0 & DKK3-2 |-<> SFRP3 & DKK3-2 $<>-<>$ SFRP3 \\
\hline 5 & DKK4 & 0 & 480 & 96 & 0 & 0 & 116 & 460 & 0 & DKK4 $\mid-<>S F R P 3$ & DKK4 <> -| SFRP3 \\
\hline 6 & DACT1 & 346 & 230 & 0 & 0 & 216 & 360 & 0 & 0 & DACT1 $<>-<>$ SFRP3 & DACT1 $\mid-<>$ SFRP3 \\
\hline 7 & DACT2 & 312 & 264 & 0 & 0 & 264 & 312 & 0 & 0 & DACT $2<>-<>$ SFRP3 & DACT $2 \mid-<>$ SFRP 3 \\
\hline 8 & DACT3 & 504 & 0 & 0 & 72 & 69 & 0 & 0 & 507 & DACT $3<>-<>$ SFRP3 & DACT3 | - | SFRP3 \\
\hline 9 & SFRP1 & 552 & 24 & 0 & 0 & 46 & 460 & 0 & 70 & SFRP1 $<>-<>$ SFRP3 & SFRP1 $\mid-<>$ SFRP3 \\
\hline 10 & SFRP2 & 480 & 0 & 0 & 96 & 96 & 480 & 0 & 0 & SFRP2 $<>-<>$ SFRP3 & SFRP2 $\mid-<>$ SFRP3 \\
\hline 11 & SFRP4 & 264 & 312 & 0 & 0 & 312 & 264 & 0 & 0 & SFRP4 |- <> SFRP3 & SFRP4 $<>-<>$ SFRP3 \\
\hline 12 & SFRP5 & 460 & 0 & 0 & 116 & 115 & 0 & 0 & 461 & SFRP5 $<>-<>$ SFRP3 & SFRP5 | - | SFRP3 \\
\hline 13 & WIF1 & 0 & 408 & 168 & 0 & 0 & 178 & 398 & 0 & WIF1 $\mid-<>$ SFRP3 & WIF1 $<>-\mid$ SFRP3 \\
\hline 14 & LEF1 & 0 & 480 & 96 & 0 & 0 & 92 & 484 & 0 & LEF1 $1-<>$ SFRP 3 & LEF1 $<>-\mid$ SFRP3 \\
\hline 15 & MYC & 0 & 456 & 120 & 0 & 0 & 134 & 442 & 0 & MYC $\mid-<>$ SFRP3 & MYC $<>-$ SFRP3 \\
\hline 16 & CCND1 & 0 & 480 & 96 & 0 & 0 & 96 & 480 & 0 & CCND1 $\mid-<>$ SFRP3 & CCND1 $<>-\mid$ SFRP3 \\
\hline 17 & CD44 & 0 & 376 & 200 & 0 & 0 & 192 & 384 & 0 & CD44 |-<> SFRP3 & CD44 <> -| SFRP3 \\
\hline \multicolumn{12}{|c|}{$S F R P 3$ activation status apropos gene evidences in Normal and Tumor samples using $\theta=\theta_{N}$ and $\theta=\theta_{T}$} \\
\hline 1 & DKK1 & 0 & 360 & 216 & 0 & 360 & 216 & 0 & 0 & DKK1 $\mid-<>$ SFRP3 & DKK1 $<>-<>$ SFRP3 \\
\hline 2 & DKK2 & 360 & 0 & 0 & 216 & 216 & 360 & 0 & 0 & DKK2 $<>-<>$ SFRP3 & DKK2 $\mid-<>$ SFRP3 \\
\hline 3 & DKK3-1 & 360 & 0 & 0 & 216 & 216 & 360 & 0 & 0 & DKK3-1 $<>-<>$ SFRP3 & DKK3-1 $\mid-<>$ SFRP3 \\
\hline 4 & DKK3-2 & 0 & 328 & 240 & 8 & 336 & 240 & 0 & 0 & DKK3-2 |- <> SFRP3 & DKK3-2 $<>-<>$ SFRP3 \\
\hline 5 & DKK4 & 0 & 480 & 96 & 0 & 0 & 116 & 460 & 0 & DKK4 $\mid-<>$ SFRP3 & DKK4 <> -| SFRP3 \\
\hline 6 & DACT1 & 346 & 230 & 0 & 0 & 216 & 360 & 0 & 0 & DACT1 $<>-<>$ SFRP3 & DACT1 $\mid-<>$ SFRP3 \\
\hline 7 & DACT2 & 24 & 0 & 288 & 264 & 264 & 312 & 0 & 0 & DACT2 $<>-\mid$ SFRP3 & DACT $2 \mid-<>$ SFRP 3 \\
\hline 8 & DACT3 & 504 & 0 & 0 & 72 & 69 & 0 & 0 & 507 & DACT3 $<>-<>$ SFRP3 & DACT3 | - | SFRP3 \\
\hline 9 & SFRP1 & 552 & 0 & 0 & 24 & 46 & 530 & 0 & 0 & SFRP1 $<>-<>$ SFRP3 & SFRP1 $\mid-<>$ SFRP3 \\
\hline 10 & SFRP2 & 480 & 0 & 0 & 96 & 96 & 480 & 0 & 0 & SFRP2 $<>-<>$ SFRP3 & SFRP2 $\mid-<>$ SFRP3 \\
\hline 11 & SFRP4 & 0 & 77 & 264 & 235 & 312 & 264 & 0 & 0 & SFRP4 <> -| SFRP3 & SFRP4 $<>-<>$ SFRP3 \\
\hline 12 & SFRP5 & 460 & 0 & 0 & 116 & 115 & 411 & 0 & 50 & SFRP5 $<>-<>$ SFRP3 & SFRP5 |- <> SFRP3 \\
\hline 13 & WIF1 & 0 & 408 & 168 & 0 & 398 & 178 & 0 & 0 & WIF1 |- <> SFRP3 & WIF1 $<>-<>$ SFRP3 \\
\hline 14 & LEF1 & 0 & 480 & 96 & 0 & 0 & 92 & 484 & 0 & LEF1 $\mid-<>$ SFRP3 & LEF1 <> -| SFRP3 \\
\hline 15 & MYC & 0 & 456 & 120 & 0 & 0 & 134 & 442 & 0 & MYC $\mid-<>$ SFRP3 & MYC $<>-\mid$ SFRP3 \\
\hline 16 & CCND1 & 0 & 480 & 96 & 0 & 0 & 96 & 480 & 0 & CCND1 $\mid-<>$ SFRP3 & CCND1 $<>-\mid$ SFRP3 \\
\hline 17 & CD44 & 0 & 376 & 200 & 0 & 384 & 192 & 0 & 0 & CD44 |- <> SFRP3 & CD44 $<>-<>$ SFRP3 \\
\hline
\end{tabular}

Table 3: SFRP3 activation status in test samples conditional on status of individual gene activation (represented by evidence in test data) in Normal and Tumor samples. Measurements are taken over summation of all predicted values across the different runs of the 2-Hold out experiment. Here the notations denote the following: a - active, $\mathrm{p}$ - passive, $\mathrm{N}$ - Normal, $\mathrm{T}$ Tumor, $g g_{I N}$ - gene-gene interaction with Normal, $g g_{I T}$ - gene-gene interaction with Tumor, $<>-$ active and $\mid$ - repressed.

interactions - the inferred interactions differ based on the choice of the threshold involved (which is a computational issue) but if prevalent, the underlying psychophysical laws remain unchanged (which is a natural phenomena irrespective of the components involved).

The network obtained by using an arbitrary value like 0.5 for labeling the gene interactions is different from those obtained using a weighted mean. There are advantages of choosing the weighted mean of the training labels for each gene - • Each gene has an individual threshold that is different from the other as the expression values are different and the discretization used to estimate a particular threshold is based on the median value of the training data for that particular gene under consideration. - The weighted mean assigns appropriate weights to the labels under consideration rather than assigning equal weights which might not represent the actual threshold. • Due to the properties mentioned in the second point, it might be expected that the weighted mean generates a sparse network in comparison to that generated using an arbitrary 
bioRxiv preprint doi: https://doi org/10.1101/015834; this version posted May 10, 2017. The copyright holder for this preprint (which was not certified by peer review) is the author/funder, who has granted bioRxiv a license to display the preprint in perpetuity. It is made available under aCC-BY-NC-ND 4.0 International license.

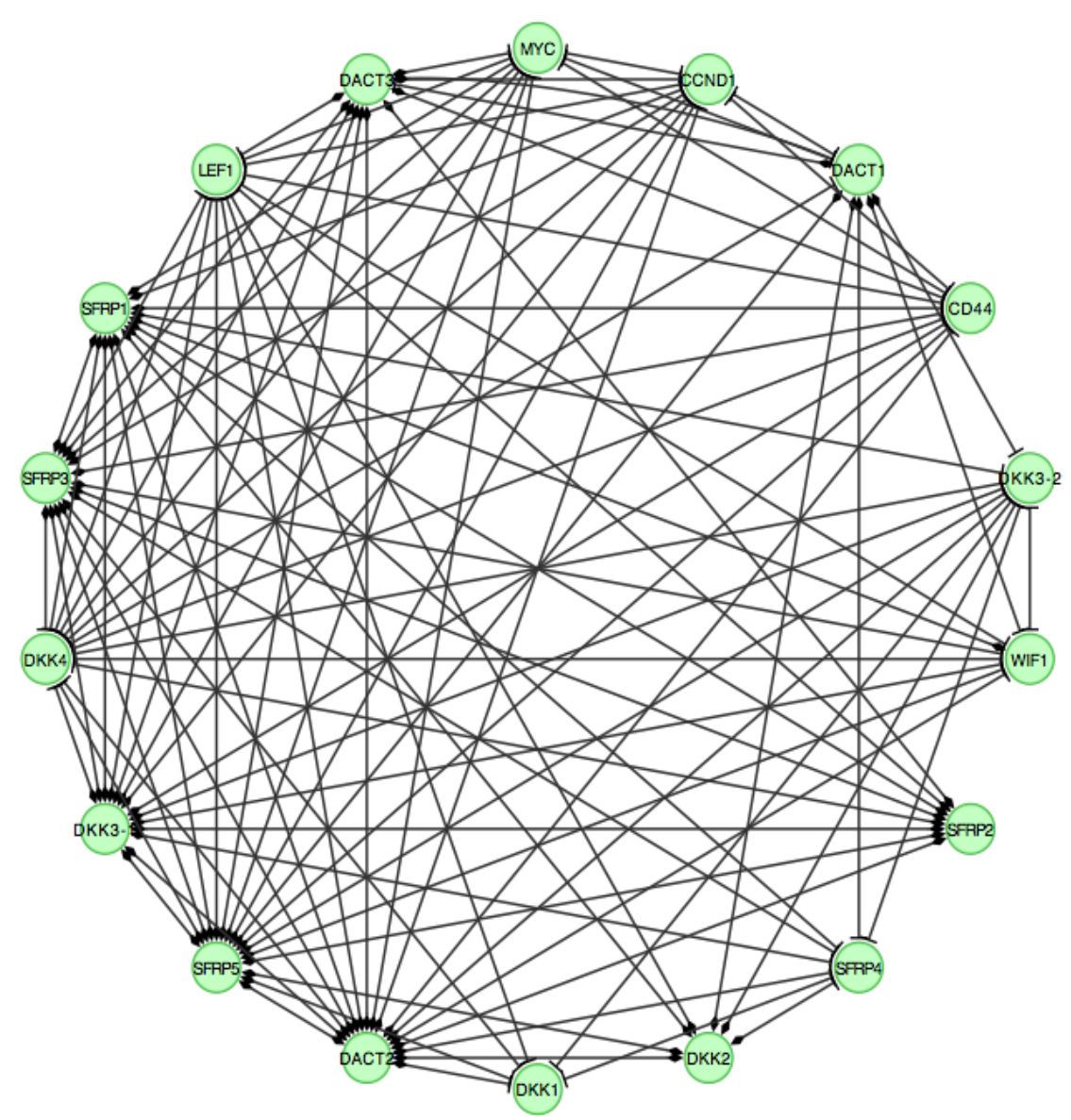

Figure 8: Gene gene interactions for normal case while using $\mathcal{M}_{P B K+E I}$ with $\theta=0.5$. Note that the effect of initialized cpt for TRCMPLX is $90 \%$ in tumorous case and $10 \%$ in normal case. Diamond $<>$ means activation and straight bar $\mid$ means repression.

value of 0.5 . - Finally, the weighted mean could reveal interactions between two genes that might be happening at different stages of time. Even though using a static model, capturing such intricate interactions is possible as will be seen later.

There is a formulation for weighted means, but the computation of the weighted mean for training samples belonging to Normal and Tumor is done 
bioRxiv preprint doi: https://doi org/10.1101/015834 this version posted May 10, 2017. The copyright holder for this preprint (which was not certified by peer review) is the author/funder, who has granted bioRxiv a license to display the preprint in perpetuity. It is made available under aCC-BY-NC-ND 4.0 International license.

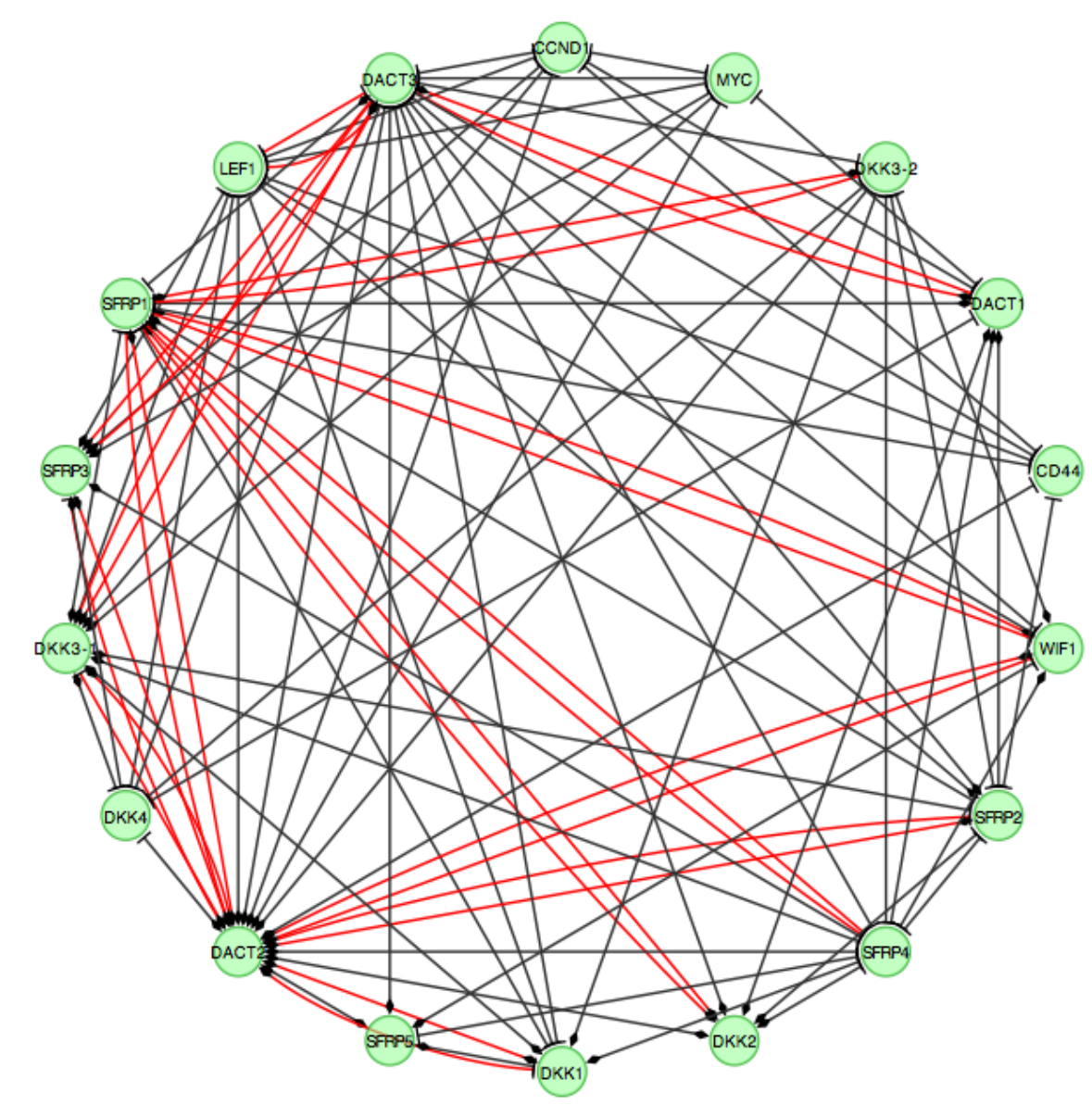

Figure 9: Gene gene interactions for normal case while using $\mathcal{M}_{P B K+E I}$ with $\theta=\theta_{N}$. Note that the effect of initialized cpt for TRCMPLX is $90 \%$ in tumorous case and $10 \%$ in normal case. Diamond $<>$ means activation and straight bar $\mid$ means repression.

separately. The separate formulations are given below -

$$
\begin{aligned}
\theta_{N} & =\frac{1 \times n_{1, N}+2 \times n_{2, N}}{(1+2) \times\left(n_{1, N}+n_{2, N}\right)} \\
\theta_{T} & =\frac{1 \times n_{1, T}+2 \times n_{2, T}}{(1+2) \times\left(n_{1, T}+n_{2, T}\right)}
\end{aligned}
$$

were, $n_{1, N}$ is the number of Normal training samples with label $1, n_{2, N}$ is the number of Normal training samples with label $2, n_{1, T}$ is the number of Tumor training samples with label 1 and $n_{1, N}$ is the number of Tumor training samples with label 2. Note that the sample labels (i,e evidence of gene expression) were discretized to passive or 1 (active or 2). 


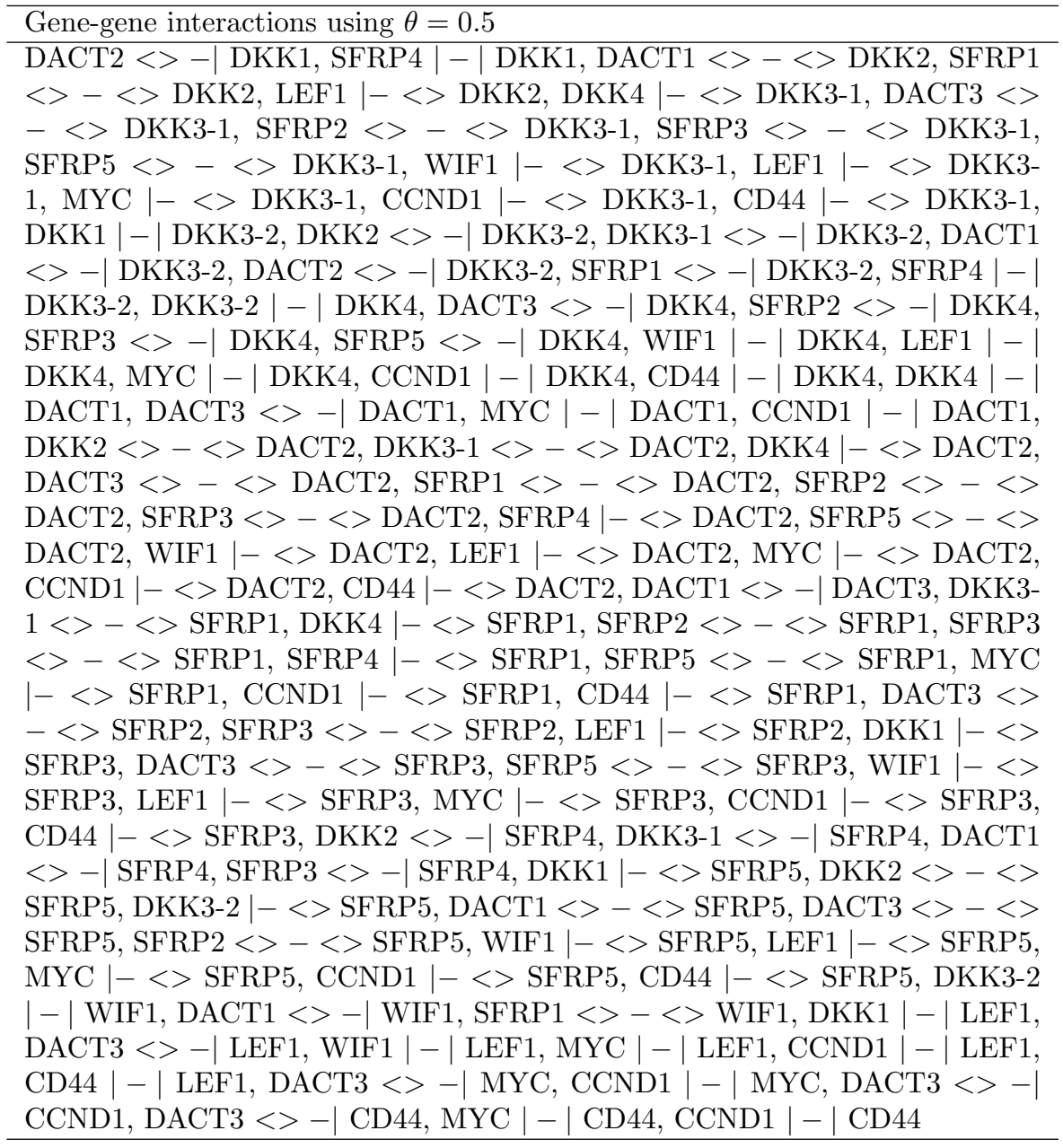

Table 4: Tabulated gene gene interactions of figure 8 using $\mathcal{M}_{P B K+E I}$ obtained in case of Normal samples. Here, the symbols represent the following $-\langle>$ activation and $|$ repression/suppression. Note that for Tumor cases, the interaction roles were found to be reversed, ie. $\langle>-|$ in normal became $\mid-\langle>$ in tumor, $|-\langle>$ in normal became $\langle>-|$ in tumor, $<>-<>$ in normal became $|-|$ in tumor and $|-|$ in normal became $<>-<>$ in tumor.

Based on the steps described in Appendix, for each gene a matrix is obtained that shows the statistics of how the status of a gene is affected conditional on the individual evidences of the remaining genes. Also, for each of the $i^{\text {th }}$ gene the averaged $\widehat{\operatorname{Pr}}_{N}\left(g_{i} \mid g_{k}\right)$ is also stored in vector $\operatorname{PggN}$. Same is done for tumor cases. These two vectors are later used to test the veracity of existence of psychophysical laws in gene-gene interaction network. Table 3 represents one such tabulation for gene $S F R P 3$. For all runs and all test samples, the following was tabulated in table 3: $a a_{N}-S F R P 3$ is active (a) when a gene is active (a) 


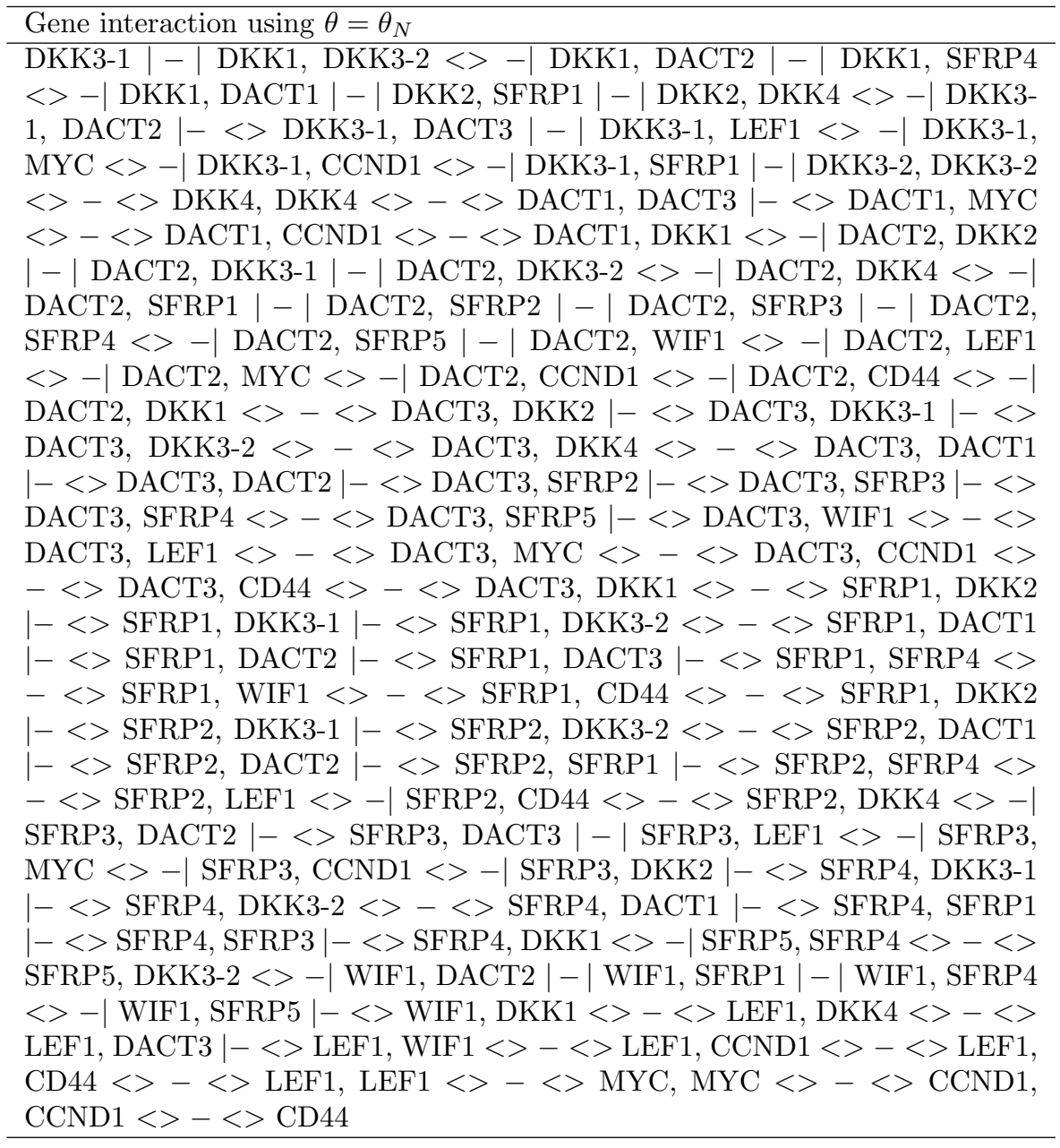

Table 5: Tabulated gene gene interactions of figure 9 using $\mathcal{M}_{P B K+E I}$ obtained in case of Normal samples. Here, the symbols represent the following $-\langle>$ activation and $|$ repression/suppression. Note that for Tumor cases, the interaction roles were found to be reversed, ie. $\langle>-|$ in normal became $\mid-\langle>$ in tumor, $|-\langle>$ in normal became $\langle>-|$ in tumor, $<>-<>$ in normal became $|-|$ in tumor and $|-|$ in normal became $<>-<>$ in tumor.

in Normal (N) sample, $a r_{N}-S F R P 3$ is active (a) when a gene is repressed (r) in Normal (N) sample, $r a_{N}-S F R P 3$ is repressed (r) when a gene is active (a) in Normal (N) sample, $r r_{N}-S F R P 3$ is repressed (r) when a gene is repressed (r) in Normal (N) sample, $a a_{T}-S F R P 3$ is active (a) when a gene is active (a) in Tumor (T) sample, $a r_{T}-S F R P 3$ is active (a) when a gene is repressed (r) in Tumor (T) sample, $p a_{T}-S F R P 3$ is repressed (r) when a gene is active (a) in Tumor (T) sample, $g g_{I N}$ - interaction of $S F R P 3$ given the gene evidence based on majority voting among $a a_{N}, a r_{N}, r a_{N}$ and $r r_{N}$ and finally, $g g_{I T}$ - 


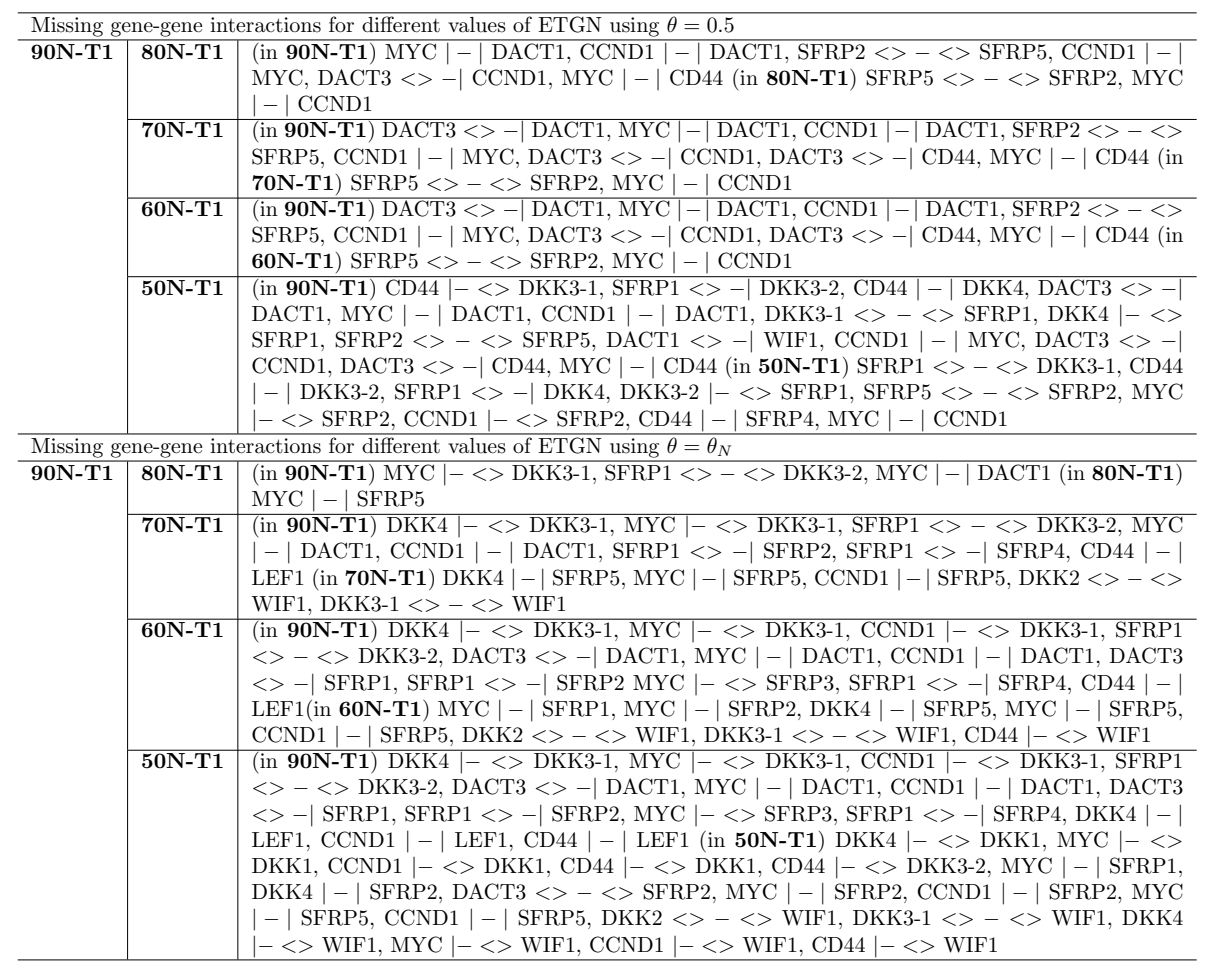

Table 6: Tabulated missing gene gene interactions of figure 8 and 9 using $\mathcal{M}_{P B K+E I}$ obtained in case of Normal samples. Interactions found in Normal samples with $80 \%, 70 \%, 60 \%$ and $50 \%$ effect that are not found with $90 \%$ and vice versa have been recorded. Here, the symbols represent the following $-\langle>$ activation and $|$ repression/suppression. Note that for Tumor cases, the interaction roles were found to be reversed, ie. $<>-\mid$ in normal became $\mid-<>$ in tumor, $\mid-<>$ in normal became $<>-\mid$ in tumor, $\langle>-<>$ in normal became $|-|$ in tumor and $|-|$ in normal became $<>-<>$ in tumor. 
interaction of $S F R P 3$ given the gene evidence based on majority voting among $a a_{T}, a r_{T}, r a_{T}$ and $r r_{T}$. The highest score among $a a_{N}, a r_{N}, r a_{N}$ and $r r_{N}$ $\left(a a_{T}, a r_{T}, r a_{T}\right.$ and $\left.r r_{T}\right)$ confirms the relation between genes using Normal (Tumor) samples. Activation (repression) for $S F R P 3$ is based on discretizing the predicted conditional probability $\operatorname{Pr}\left(S F R P 3=\right.$ active $\mid g_{j}$ evidence $)$ as $\geq \theta$ $(<\theta)$. Activation (repression) for a particular gene evidence $g_{j}$ is done using discrete evidence. In table 3 , under the columns $g g_{I N}$ and $g g_{I T},\langle>$ implies the gene is active and $\mid$ implies the gene is repressed or passive.

\section{Gene-gene interaction network when $\theta=0.5$}

Considering only reversible interactions, in table 3 it was found that evidence for $D K K 1$ and $D K K 4$ show similar repression behaviour as the standard genes $W I F 1, L E F 1, M Y C, C C N D 1$ and $C D 44$ in Normal (Tumor) test samples. Only, SFRP5 and DACT3 in Normal (Tumor) test samples shows activation (repression). Conditional on the observed activation status of the genes mentioned above, $S F R P 3$ shows activated (repressed) state in Normal (Tumor) test samples. $S F R P 3$ showed behaviour similar to $S F R P-1 / 2 / 5$. Since it is known that the activation status of the latter is influenced by epigenetic factors, $S F R P 3$ might also be influenced by epigenetic factors.

Irreversible interactions present in table 3 are deleted as they do not provide concrete information regarding the functional roles of the genes in normal and tumor cases. This attributes to one of the following facts (1) noise that corrupts prediction values as can be seen in the columns of $a a_{N}\left(a a_{T}\right), a r_{N}\left(a r_{T}\right), r a_{N}$ $\left(r a_{T}\right)$ and $r r_{N}\left(r r_{T}\right)$ or (2) other multiple genes might be interacting along with $S F R P 3$ in a combined manner and it is not possible to decipher the relation between $S F R P 3$ and other genes. This calls for investigation of prediction of $S F R P 3$ status conditional on joint evidences of two or more genes (a combinatorial problem with a search space order of $2^{17}-17$, which excludes 17 cases of individual gene evidences which have already been considered here). Incorporating multiple gene evidences is not a problem while using Bayesian network models as they are designed to compute conditional probabilities given joint evidences also (except at the cost of high computational time).

It is evident that an arbitrary value of $\theta=0.5$ will not generate appropriate networks. This is due to the fact that 0.5 does not encode the biological knowledge of thresholding while using discretization. To over come this, a weighted mean is employed as shown below.

Gene-gene interaction network when $\theta=\theta_{N}^{S F R P 3}$

While employing the weighted mean as the threshold to discretize $\operatorname{Pr}(S F R P 3$ $=$ active $\mid g_{j}$ evidence), the $S F R P 3$ gene evidences that constitutes the test data are used. See step 5.a.iii in Appendix. Note that the test evidences for SFRP3 are used for two purpose (1) to discretize $\operatorname{Pr}\left(S F R P 3=\right.$ active $\mid g_{j}$ evidence) as discussed above and (2) to compute the probability of activation status of another gene conditional on evidence for $S R F P 3$, i.e $\operatorname{Pr}\left(g_{j}=\right.$ active $\mid S F R P 3$ evidence). Why to use test evidence or labels to compute weighted mean? Since the test evidence for a gene (i.e the discretized label) has been derived using the median computed on the corresponding training data for the same gene, it absolutely fine to use the discretized test labels to further compute the 
weighted mean. This is because the median of gene expression is a value which is much higher than the probability value of 1 and cannot be used to discretize a predicted conditional probability value. Also, estimating the density estimates from a small population of gene expression values has its own weakness. To converge on a plausible realistic value the discretized test samples can be used to estimate a weighted mean which represents the summary of the distribution of the discretized values. This weighted mean of $S F R P 3$ test samples then discretizes $\operatorname{Pr}\left(S F R P 3=\right.$ active $\mid g_{j}$ evidence $)$ according to the inherently represented summary. More realistic estimates like kernel density estimates could also be used.

In comparison to the interactions derived using $\theta=0.5$ in table 3 it was found that a more restricted list of $D K K 4, D A C T-2 / 3, L E F 1, M Y C$ and $C C N D 1$ showed reversible behaviour with SFRP3 using the weighted mean. This reduction in the reversible interactions is due to the fact that the weighted mean carries an idiosyncrasy of the test label data distribution and is more restricted in comparison to the use of 0.5 value that was arbitrarily chosen. Finally, using the proposed weighted mean reveals more than one interaction between two genes. These interactions point to important hidden biological phenomena that require further investigation in the form of wet lab experiments and the ensuing in silico analysis. It also points to the fact that a particular gene may be showing different behaviour at different times in the network while interacting with multiple genes. An example of this will be addressed later. Again, dynamic models will bring more clarity to the picture. Table 3 shows these interactions using $\theta \in\left\{0.5, \theta_{N}, \theta_{T}\right\}$.

\subsection{Inferring gene-gene interaction network}

Next, after the construction of gene-gene interactions, it is necessary to infer the network. The inference of the estimated gene-gene interactions network is based on explicitly reversible roles in Normal and Tumor test samples. This means that only those interactions are selected which show the following property $-g_{j}<>-<>g_{i}$ in Normal if and only if $g_{j}|-| g_{i}$ in Tumor, $g_{j}<>-\mid g_{i}$ in Normal if and only if $g_{j} \mid-<>g_{i}$ in Tumor, $g_{j} \mid-<>g_{i}$ in Normal if and only if $g_{j}<>-\mid g_{i}$ in Tumor and finally, $g_{j}|-| g_{i}$ in Normal if and only if $g_{j}<>-<>g_{i}$. This restricts the network to only reversible gene-gene interactions in Normal and Tumor cases. Note that an interaction $g_{j} \mathcal{I} \mathcal{R} g_{i}\left(g_{i} \mathcal{I} \mathcal{R} g_{j}\right)$ is depicted by $\operatorname{Pr}\left(g_{i} \mid g_{j}\right)\left(\operatorname{Pr}\left(g_{j} \mid g_{i}\right)\right)$.

Reversibility helps in tracking the behaviour of gene-gene interaction in both normal and tumor case simultaneously and thus give more weight to confirmatory results. Irreversible reactions here mean that the state of activation of a gene in both normal and tumor sample remains invariant given the evidence of the other gene in the gene-gene interaction. This helps in eliminating the interactions that might not be happening at all from biological perspective. To confirm the computational results wet lab experiments are needed. See table 3 for reversible and irreversible interactions.

Next, duplicate interactions are removed from the network for normal samples. This is repeated for the network based on tumor samples also. This is 
Deviation study for $S F R P 3$ and $M Y C$ for normal case

\begin{tabular}{c|c|c|c|c|c}
\hline$\beta$ & $\Delta \beta$ & $\frac{\Delta \beta}{\beta}$ & $\log \left(1+\frac{\Delta \beta}{\beta}\right)$ & $\begin{array}{c}\Delta \gamma \\
\operatorname{Pr}(S F P R 3 \mid M Y C)\end{array}$ & $\operatorname{Pr}(M Y C \mid S F P R 3)$ \\
\hline 0.8 & 0.1 & 0.125 & 0.117783 & 0.003014287 & 0.00324456 \\
0.7 & 0.1 & 0.1428571 & 0.1335314 & 0.002766111 & 0.00324456 \\
0.6 & 0.1 & 0.1666667 & 0.1541507 & 0.002504868 & 0.00324456 \\
0.5 & 0.1 & 0.2 & 0.1823216 & 0.002228110 & 0.00324456 \\
\hline 0.8 & 0.1 & 0.125 & 0.117783 & 0.010513376 & 0.01297824 \\
0.7 & 0.2 & 0.2857143 & 0.2513144 & 0.007499089 & 0.00973368 \\
0.6 & 0.3 & 0.5 & 0.4054651 & 0.004732978 & 0.00648912 \\
0.5 & 0.4 & 0.8 & 0.5877867 & 0.002228110 & 0.00324456 \\
\hline
\end{tabular}

Table 7: Deviation study for $\operatorname{Pr}(S F R P 3 \mid M Y C)$ and $\operatorname{Pr}(M Y C \mid S F R P 3)$ for normal case

achieved by removing one of the interactions from the following pairs $\left(g_{j}<>\right.$ $-<>g_{i}$ and $\left.g_{i}<>-<>g_{j}\right),\left(g_{j}<>-\mid g_{i}\right.$ and $\left.g_{i} \mid-<>g_{j}\right),\left(g_{j} \mid-<>g_{i}\right.$ and $\left.g_{i}<>-\mid g_{j}\right)$ and $\left(g_{j}|-| g_{i}\right.$ and $\left.g_{i}|-| g_{j}\right)$. This process is done to remove redundant interactions that are recorded via steps mentioned in construction of gene-gene interaction network. Figure 8 shows one such network after complete network construction, interaction labeling, consideration of reversible interactions and removal of duplicate interactions using Normal test samples with ETGN of $90 \%$ in $\mathcal{M}_{P B K+E I}$. For the case of Tumor test samples with ETGN $90 \%$ in $\mathcal{M}_{P B K+E I}$, only the reversal of interactions need to be done. Table 4 and 5 represents these interactions in figures 8 and 9 in a tabulated form, respectively.

Finally, different networks were generated by varying the effect of TRCMPLX (ETGN) and compared for the normal test samples. Table 6 represents the different interactions that were preserved in network from ETGN $90 \%$ with respect to networks obtained from ETGN with values of $80 \%, 70 \%, 60 \%$ and $50 \%$. It was found that most of the genetic interactions depicted in figures 8 and 9 were found to be preserved across the different variations in ETGN as shown in table 6. Out of the total $n$ genes which construct a fully connected graph of $\frac{n \times(n-1)}{2}$, it was observed that lesser number of interconnections were preserved. This preservation indicates towards the robustness of the genetic contributions in the Wnt signaling pathway in both normal and tumor test samples. Note that these observations are made from static models and dynamic models might reveal greater information.

\section{Results and discussion on observations $2 \& 3$}

\subsection{Logarithmic-power deviations in prediction of gene-gene interactions}

In the previous section, it was found that some of the interactions remain preserved as there was change in the affect of transcription complex. The first 
Deviation study for $S F R P 3$ and $M Y C$ for tumor case

\begin{tabular}{c|c|c|c|c|c}
\hline$\beta$ & $\Delta \beta$ & $\frac{\Delta \beta}{\beta}$ & $\log \left(1+\frac{\Delta \beta}{\beta}\right)$ & $\Delta \gamma$ & $\Delta \gamma$ \\
& & & & $\operatorname{Pr}(S F P R 5 \mid M Y C)$ & $\operatorname{Pr}(M Y C \mid S F P R 5)$ \\
\hline 0.8 & 0.1 & 0.125 & 0.117783 & -0.006463410 & $0.000000 \mathrm{e}+00$ \\
0.7 & 0.1 & 0.1428571 & 0.1335314 & -0.006967724 & $5.551115 \mathrm{e}-17$ \\
0.6 & 0.1 & 0.1666667 & 0.1541507 & -0.007515486 & $-5.551115 \mathrm{e}-17$ \\
0.5 & 0.1 & 0.2 & 0.1823216 & -0.008112496 & $0.000000 \mathrm{e}+00$ \\
\hline 0.8 & 0.1 & 0.125 & 0.117783 & -0.029059115 & $0.000000 \mathrm{e}+00$ \\
0.7 & 0.2 & 0.2857143 & 0.2513144 & -0.022595705 & $0.000000 \mathrm{e}+00$ \\
0.6 & 0.3 & 0.5 & 0.4054651 & -0.015627982 & $-5.551115 \mathrm{e}-17$ \\
0.5 & 0.4 & 0.8 & 0.5877867 & -0.008112496 & $0.000000 \mathrm{e}+00$ \\
\hline
\end{tabular}

Table 8: Deviation study for $\operatorname{Pr}(S F R P 3 \mid M Y C)$ and $\operatorname{Pr}(M Y C \mid S F R P 3)$ for tumor case

observation of this work was that deviations in the activity of the transcription complex followed a logarithmic-power psychophysical law. The manifestation of these laws at transcriptional levels can be attributed to the fold changes in $\beta$-catenin levels and the prevalence of Weber's law observed by Goentoro and Kirschner (2009). In this perspective, it would be interesting to observe if these laws are prevalent among the gene-gene interactions in the network or not.

Case: $<>-\mid$ or $\mid-<>$ with $\theta=\theta_{N}$

In Sinha (2014), the unknown behaviour of SFRP3 in the Wnt pathway has been revealed slightly using computational causal inference. In figure 8 , $S F R P 3$ shows preservation in the network and it's interaction with other genetic factors involved in the model proposed in Sinha (2014) has been depicted. In one such paired interaction between $S F R P 3$ and $M Y C, S F R P 3$ showed activation (repression) and $M Y C$ showed repression (activation) in normal (tumor) samples. As the change in the effect of transcription complex was induced by changing the initially assigned cpt values for TRCMPLX node, the deviations in the prediction of the gene-gene interaction network was observed to follow the logarithmic-power law crudely. What this means is that deviations or fold changes might also be prevalent at the gene-gene interaction level due to the upstream fold changes in $\beta$-catenin that induces transcriptional activity. More specifically, the deviation in the joint interaction that is represented by the degree of belief via the conditional probability of status of one gene given the evidence or activation status regarding another gene i.e $\operatorname{Pr}\left(g_{i} \mid g_{j}\right)$, is influenced by the fold changes upstream of the pathway and thus exhibit similar psychophysical laws.

Table 7 and 8 show these deviations in the prediction of the interactions for both the normal and the tumor cases. The tables show how deviations are affected when the changes in the effect of the transcription complex are done at constant and incremental rate. To summarize the results in these tables, graphs 
were plotted in figures 10 for $\operatorname{Pr}(S F R P 3 \mid M Y C)$ (constant deviations), 11 for $\operatorname{Pr}(M Y C \mid S F R P 3)$ (constant deviations), 12 for $\operatorname{Pr}(S F R P 3 \mid M Y C)$ (incremental deviations) and 11 for $\operatorname{Pr}(M Y C \mid S F R P 3)$ (incremental deviations).

Considering figure 10, when deviations are constant in both Weber and Bernoulli formulation, the deviations in the prediction of $\operatorname{Pr}(S F R P 3 \mid M Y C)$ is observed to be logarithmic in the normal samples (apropos the Weber and Bernoulli deviations represented by green and cyan curves). Deviation in predictions are depicted by the red (blue) curves for normal (tumor) samples. Such a behaviour is not observed for $\operatorname{Pr}(M Y C \mid S F R P 3)$ as is depicted in figure 11 . Note that the interaction for $S F R P 3$ given $M Y C$ was observed to be reversible in normal and tumor cases. But this is not so with the interaction for $M Y C$ given $S F R P 3$. It might be expected that the non conformance of logarithmicpower law for $\operatorname{Pr}(M Y C \mid S F R P 3)$ may be due to the non preservation/existence of the interaction of $M Y C$ given $S F R P 3$. This is so because $\operatorname{Pr}(S F R P 3 \mid M Y C)$ depicts a reversible $S F R P 3<>-\mid M Y C(M Y C<>-\mid S F R P 3)$ in the network on normal (tumor) samples, while $\operatorname{Pr}(M Y C \mid S F R P 3)$ does not depict a reversible $M Y C \mid-<>S F R P 3(M Y C|-| S F R P 3)$ in the network on normal (tumor) samples.

Similar behaviour was observed in the case of incremental deviations as depicted in figures 12 and 13 . Analysis of the behaviour of other gene-gene interactions showing $<>-\mid$ or $\mid-<>$ can be observed in a similar way and can be produced by executing the $\mathrm{R}$ code in Weber_Fechner_law.r provided in Google drive https://drive.google.com/folderview?id=0B7Kkv8wlhPU-T05wTTNodWNydjA\&usp= sharing. Note that plots need manual axis and title adjustments. Some of the plot results has been compressed in the zip file titled Results-2015.zip.

Case: $|-|$ or $<>-<>$ with $\theta=\theta_{N}$

Again, as pointed out in Sinha (2014), the unknown behaviour of SFRP2 in the Wnt pathway has been captured using computational causal inference. In figure 9, $S F R P 2$ shows preservation in the network and it's interaction with other genetic factors involved in the model proposed in Sinha (2014) has been depicted. In one such paired interaction between $S F R P 2$ and $C D 44$, both showed repression (activation) in normal (tumor) samples. As the change in the effect of transcription complex was induced via sensitizing the initially assigned cpt values, the deviations in the prediction of the gene-gene interaction network was observed to follow the logarithmic-power law crudely.

Table 9 and 10 show these deviations in the prediction of the interactions for both the normal and the tumor cases. The tables show how deviations are affected when the changes in the effect of the transcription complex are done at constant and incremental level. To summarize the results in these tables, graphs were plotted in figures 14 for $\operatorname{Pr}(S F R P 2 \mid C D 44)$ (constant deviations), 15 for $\operatorname{Pr}(C D 44 \mid S F R P 2)$ (constant deviations), 16 for $\operatorname{Pr}(S F R P 2 \mid C D 44)$ (incremental deviations) and 15 for $\operatorname{Pr}(C D 44 \mid S F R \overline{P 2})$ (incremental deviations).

Considering figure 14, when deviations are constant in both Weber and Bernoulli formulation, the deviations in the prediction of $\operatorname{Pr}(S F R P 2 \mid C D 44)$ is observed to be logarithmic in the normal samples (apropos the Weber and Bernoulli deviations represented by green and cyan curves). Deviation in pre- 


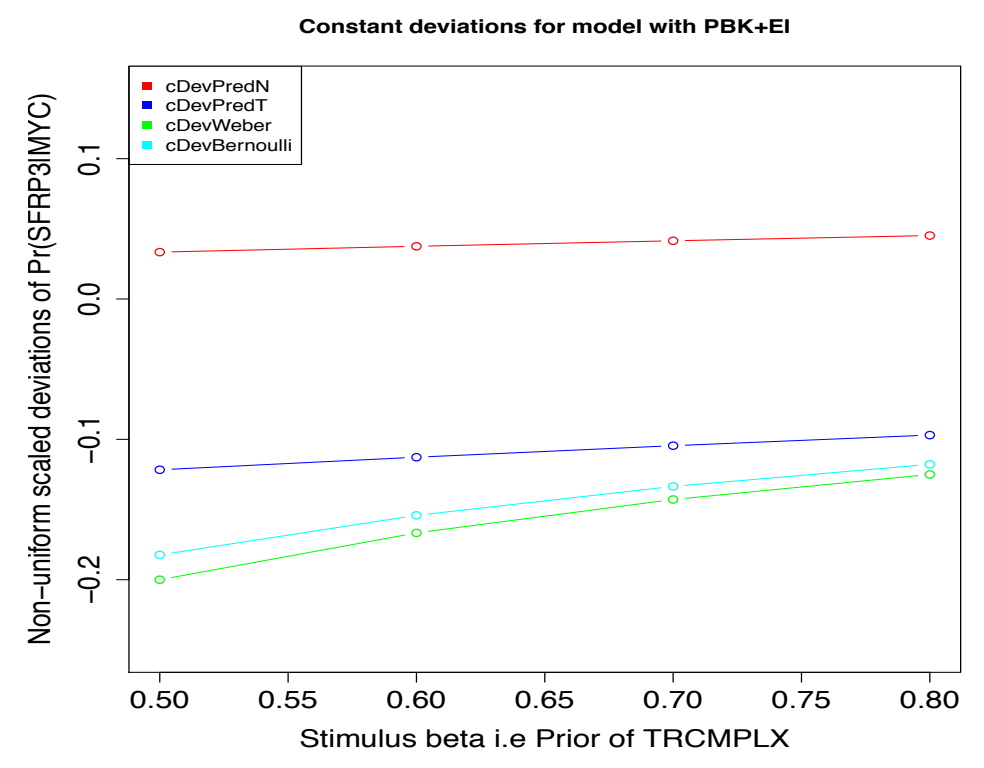

Figure 10: Constant deviations in $\beta$ i.e ETGN and corresponding deviations in $\operatorname{Pr}(S F R P 3 \mid M Y C)$ for both normal and tumor test samples. Corresponding Weber and Bernoulli deviations were also recorded. Note that the plots and the y-axis depict scaled deviations to visually analyse the observations. The model used is $\mathcal{M}_{P B K+E I}$. Red - deviation in $\operatorname{Pr}(S F R P 3 \mid M Y C)$ in Normal case using Weber's law, Blue - deviation in $\operatorname{Pr}(S F R P 3 \mid M Y C)$ in Tumor using Weber's law, Green - constant deviation in Webers law, Cyan - constant deviation in Bernoullis law.

dictions are depicted by the red (blue) curves for normal (tumor) samples. Such a behaviour is not observed for $\operatorname{Pr}(C D 44 \mid S F R P 2)$ as is depicted in figure 15. Even though $\operatorname{Pr}(C D 44 \mid S F R P 2)$ was computationally estimated through a model, the interaction for $C D 44$ given $S F R P 2$ was not observed in both normal and tumor cases while the interaction for $S F R P 2$ given $C D 44$ was observed to be reversible. This points to a crucial fact that the interactions interpreted from conditional probabilities are not always two sided. Thus the interpretation for $\operatorname{Pr}\left(g_{i} \mid g_{j}\right)$ is investigated in both directions as $g_{i} \mathcal{I} \mathcal{R} g_{j}$ and $g_{j} \mathcal{I} \mathcal{R} g_{i}$ to get a full picture. Not that the results are wrong, but all angles of interpretations need to be investigated to get the picture between any two genes. Similar behaviour was observed in the case of incremental deviations as depicted in figures 16 and 17. Note that graph for incremental deviation in $\operatorname{Pr}(C D 44 \mid S F R P 2)$ is just a cumulative effect and does not state anything about the logarithmic law.

Finally, note that the predicted conditional probability a gene $i$ given evidence for gene $j$ does not change but the inferred gene-gene interactions do change depending on the choice of the threshold. These changes are depicted in the figures 8 (table 4 )and 9 (table 5). Dual interactions were inferred using the weighted mean as a discretization factor, as is shown next. These are dual 


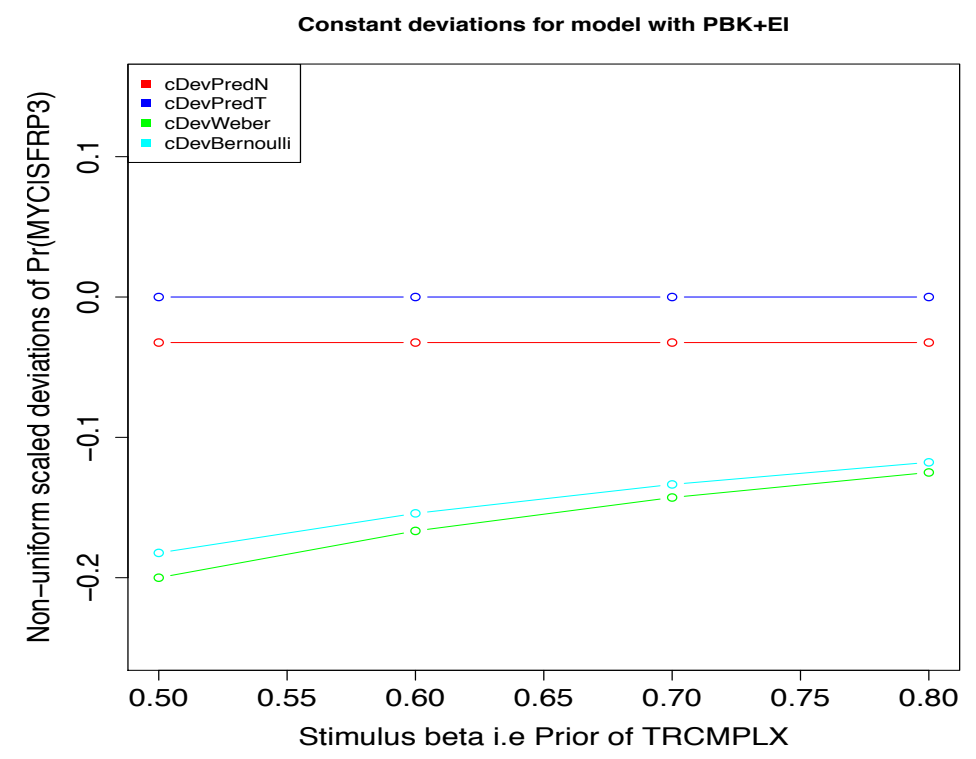

Figure 11: Same as figure 10 but for $\operatorname{Pr}(M Y C \mid S F R P 3)$.

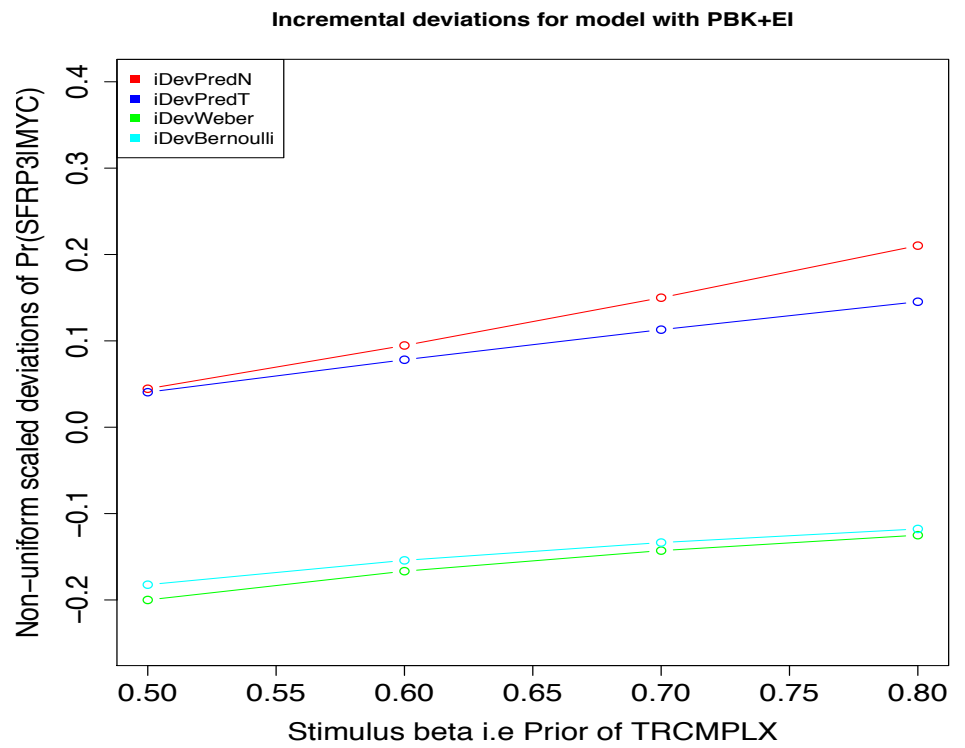

Figure 12: Same as figure 10 but for $\operatorname{Pr}(S F R P 3 \mid M Y C)$. Instead of constant deviations, incremental deviations are represented. 


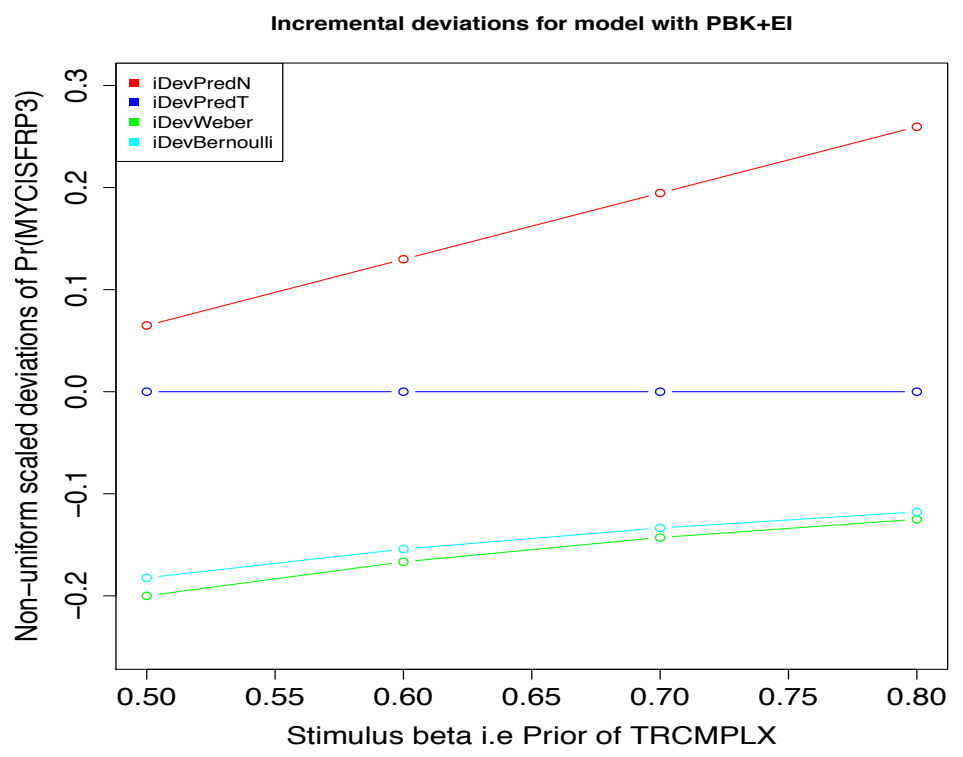

Figure 13: Same as figure 10 but for $\operatorname{Pr}(M Y C \mid S F R P 3)$. Instead of constant deviations, incremental deviations are represented.

Deviation study for $S F R P 2$ and $C D 44$ for normal case

\begin{tabular}{c|c|c|c|c|c}
\hline$\beta$ & $\Delta \beta$ & $\frac{\Delta \beta}{\beta}$ & $\log \left(1+\frac{\Delta \beta}{\beta}\right)$ & $\Delta \gamma$ & $\Delta \gamma$ \\
& & & & $\operatorname{Pr}(S F R P 2 \mid C D 44)$ & $\operatorname{Pr}(C D 44 \mid S F R P 2)$ \\
\hline 0.8 & 0.1 & 0.125 & 0.117783 & -0.0007505445 & 0.0002943409 \\
0.7 & 0.1 & 0.1428571 & 0.1335314 & -0.0009398116 & 0.0002943409 \\
0.6 & 0.1 & 0.1666667 & 0.1541507 & -0.0011360011 & 0.0002943409 \\
0.5 & 0.1 & 0.2 & 0.1823216 & -0.0013397022 & 0.0002943409 \\
\hline 0.8 & 0.1 & 0.125 & 0.117783 & -0.004166059 & 0.0011773636 \\
0.7 & 0.2 & 0.2857143 & 0.2513144 & -0.003415515 & 0.0008830227 \\
0.6 & 0.3 & 0.5 & 0.4054651 & -0.002475703 & 0.0005886818 \\
0.5 & 0.4 & 0.8 & 0.5877867 & -0.001339702 & 0.0002943409 \\
\hline
\end{tabular}

Table 9: Deviation study for $\operatorname{Pr}(S F R P 2 \mid C D 44)$ and $\operatorname{Pr}(C D 44 \mid S F R P 2)$ for normal case

interactions are marked in red colour in figure 9.

Case: Dual interactions with $\theta=\theta_{N}$

The dual interactions revealed using weighted means indicate an important phenomena between any two genes. These interactions reveal that gene activation interplay might not always be constant for normal (tumour) samples. These in silico observations imply that a gene that was found to be actively 
Deviation study for $S F R P 2$ and $C D 44$ for tumor case

\begin{tabular}{c|c|c|c|c|c}
\hline$\beta$ & $\Delta \beta$ & $\frac{\Delta \beta}{\beta}$ & $\log \left(1+\frac{\Delta \beta}{\beta}\right)$ & $\Delta \gamma$ & $\Delta \gamma$ \\
& & & $\operatorname{Pr}(S F R P 2 \mid C D 44)$ & $\operatorname{Pr}(C D 44 \mid S F R P 2)$ \\
\hline 0.8 & 0.1 & 0.125 & 0.117783 & 0.02291329 & 0.01491512 \\
0.7 & 0.1 & 0.1428571 & 0.1335314 & 0.02132802 & 0.01491512 \\
0.6 & 0.1 & 0.1666667 & 0.1541507 & 0.01962443 & 0.01491512 \\
0.5 & 0.1 & 0.2 & 0.1823216 & 0.01779600 & 0.01491512 \\
\hline 0.8 & 0.1 & 0.125 & 0.117783 & 0.08166175 & 0.05966047 \\
0.7 & 0.2 & 0.2857143 & 0.2513144 & 0.05874846 & 0.04474535 \\
0.6 & 0.3 & 0.5 & 0.4054651 & 0.03742044 & 0.02983024 \\
0.5 & 0.4 & 0.8 & 0.5877867 & 0.01779600 & 0.01491512 \\
\hline
\end{tabular}

Table 10: Deviation study for $\operatorname{Pr}(S F R P 2 \mid C D 44)$ and $\operatorname{Pr}(C D 44 \mid S F R P 2)$ for tumor case

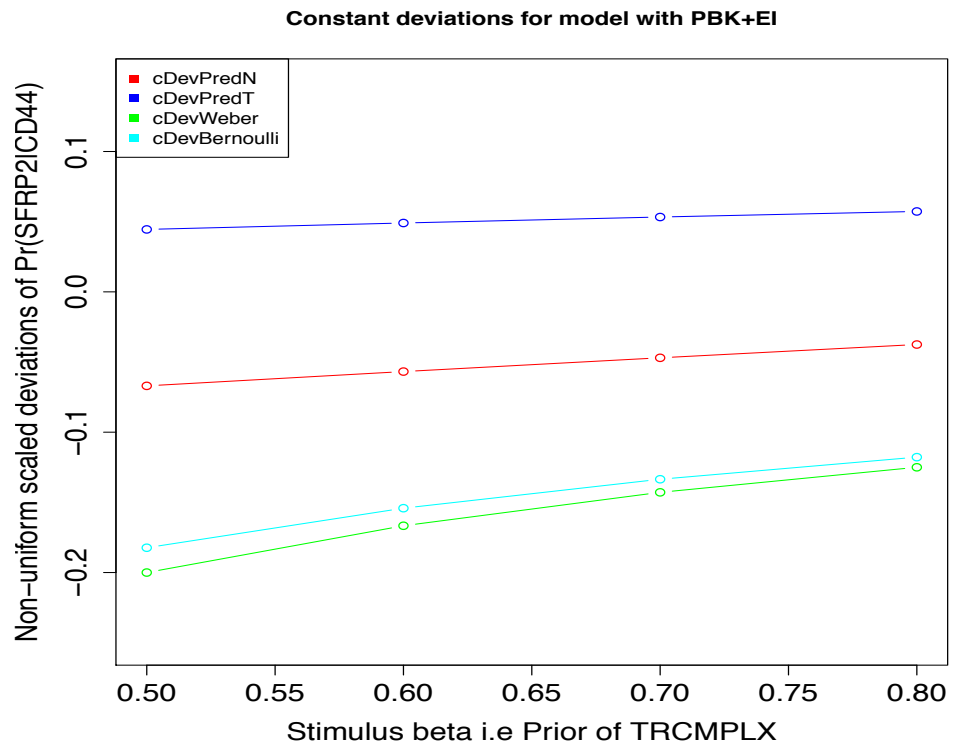

Figure 14: Constant deviations in $\beta$ i.e ETGN and corresponding deviations in $\operatorname{Pr}(S F R P 2 \mid C D 44)$ for both normal and tumor test samples. Corresponding Weber and Bernoulli deviations were also recorded. Note that the plots and the y-axis depict scaled deviations to visually analyse the observations. The model used is $\mathcal{M}_{P B K+E I}$. Red - deviation in $\operatorname{Pr}(S F R P 2 \mid C D 44)$ in Normal case using Weber's law, Blue - deviation in $\operatorname{Pr}(S F R P 2 \mid C D 44)$ in Tumor using Weber's law, Green - constant deviation in Webers law, Cyan - constant deviation in Bernoullis law.

expressed in normal sample might reverse activity at some stage or the other 
bioRxiv preprint doi: https://doi.org/10.1101/015834; this version posted May 10,2017 . The copyright holder for this preprint (which was not certified by peer review) is the author/funder, who has granted bioRxiv a license to display the preprint in perpetuity. It is made available under aCC-BY-NC-ND 4.0 International license.

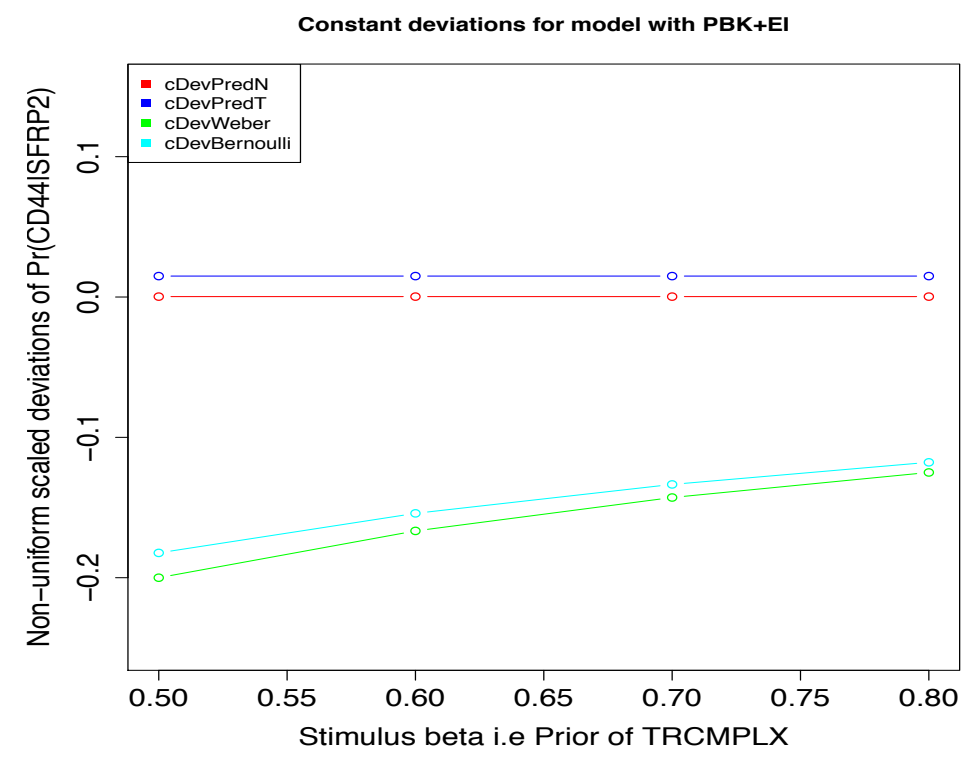

Figure 15: Same as figure 14 but for $\operatorname{Pr}(C D 44 \mid S F R P 2)$.

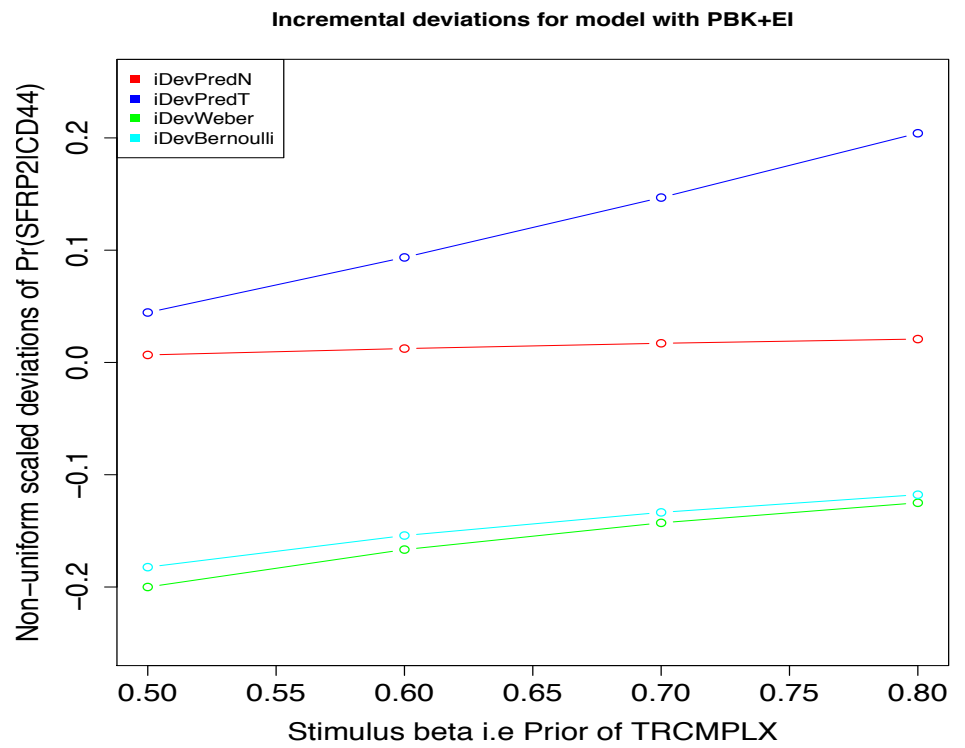

Figure 16: Same as figure 14 but for $\operatorname{Pr}(S F R P 2 \mid C D 44)$. Instead of constant deviations, incremental deviations are represented. 


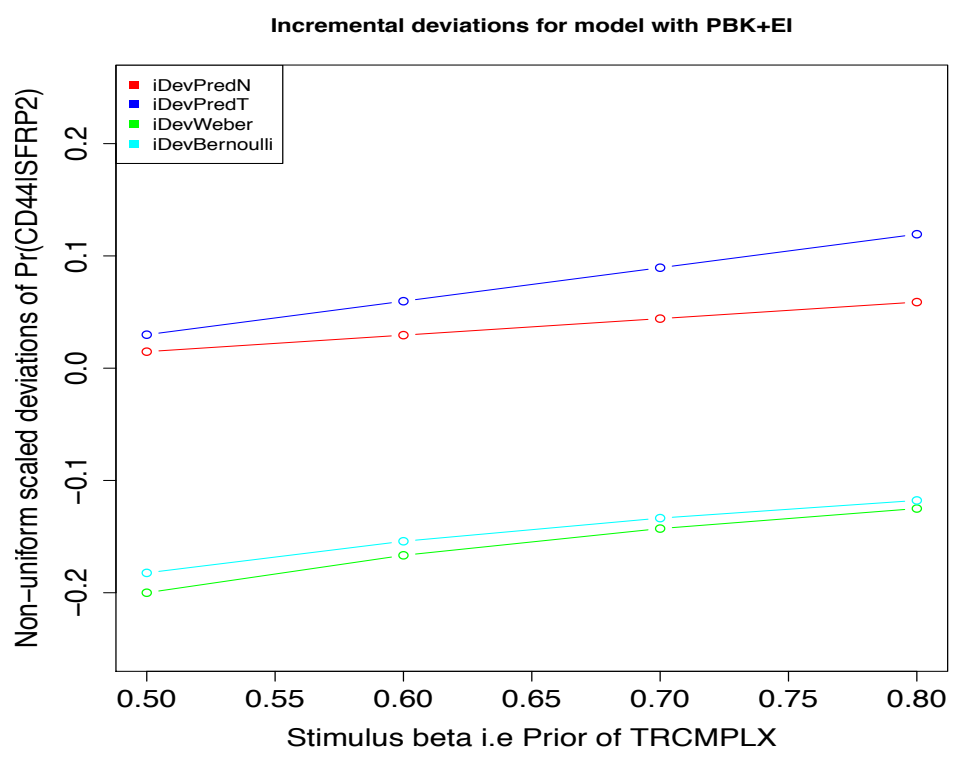

Figure 17: Same as figure 15 but for $\operatorname{Pr}(C D 44 \mid S F R P 2)$. Instead of constant deviations, incremental deviations are represented.

of the other dual interactions can be done in the same way. Results for other interactions are available but not presented here.

Also, a point to be observed is that the weighted means show much more crisp discretization during inference of gene-gene interaction in comparison to use of an arbitrary value of 0.5 . To determine this distinction between the inferred gene-gene interactions obtained via weighted threshold and the arbitrary threshold of 0.5 , the receiver operator curves (ROC) along with its corresponding area under the curve (AUC) are plotted. The ROCs are plotted using the discretized predicted values and the discretized labels obtained using the thresholds (computed from the training data) on the test data. The ROC graphs and their respective AUC values indicate how the predictions on the test data behaved under different values assigned to the TRCMPLX while training. Ideally, high values of AUC and steepness in ROC curve indicate good quality results. Finally, two sample Kolmogorov-Smirnov (KS) test was employed to measure the statistical significance between the distribution of predictions. If the cumulative distributions are not similar the KS test returns a small p-value. This small p-value indicates the existing statistical significance between the distributions under consideration.

Finally the ROC plots and AUC values for dual gene-gene interactions are also plotted and KS test is conducted to find the existence of statistical significance if any. These reveal the significance of existence of dual interactions in the signaling pathway which might not have been revealed using the arbitrary 

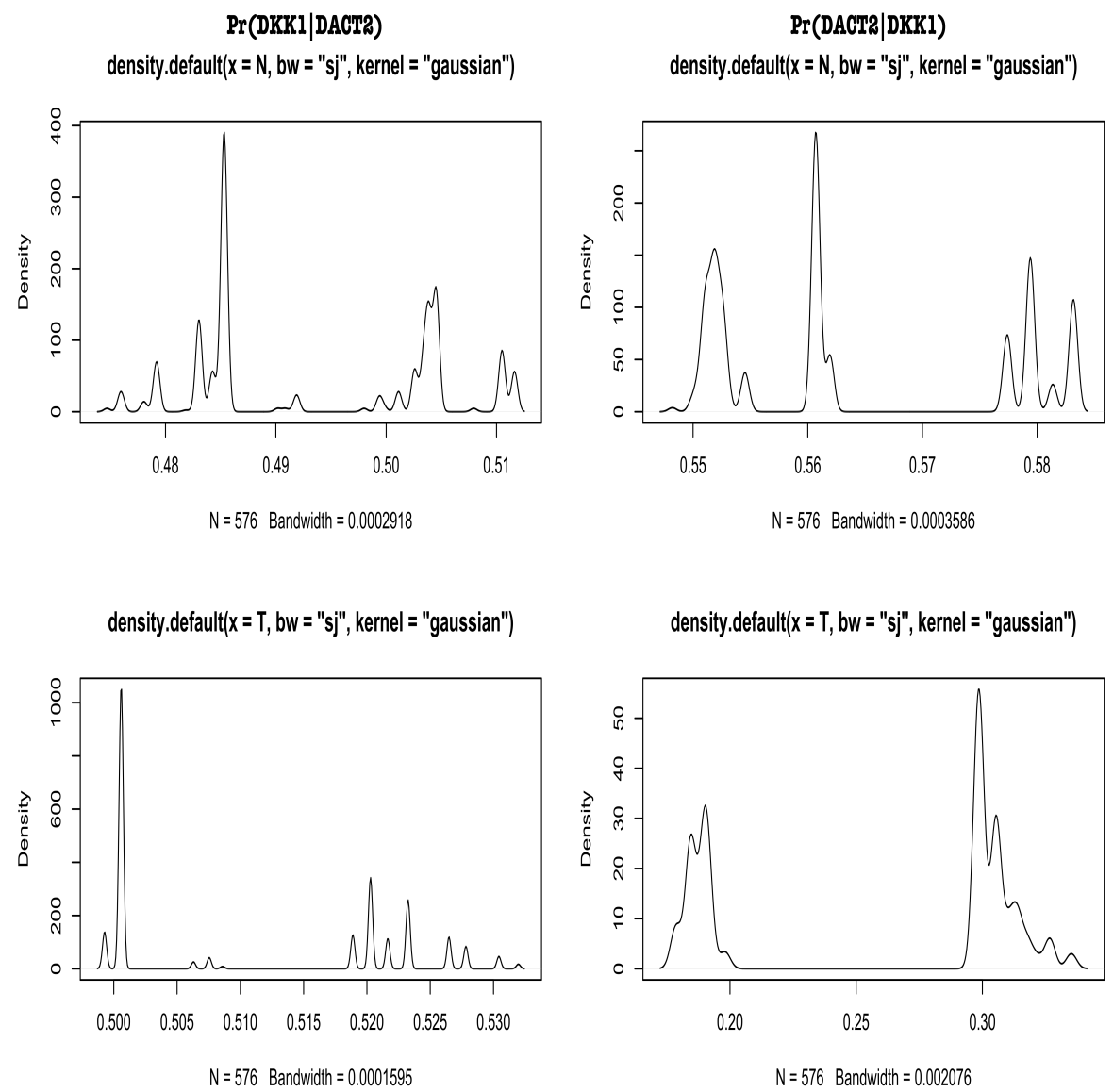

Figure 18: Kernel density estimates for predicted $\operatorname{Pr}(D K K 1 \mid D A C T 2)$ and $\operatorname{Pr}(D A C T 2 \mid D K K 1)$ in Normal and Tumor cases. Gaussian kernel is used for smoothing the density estimate. The bandwidth of the kernel is selected using the pilot estimation of derivative as proposed by Sheather and Jones (1991) and implemented in $R$ programming language.

threshold value of 0.5. Plots are made using functions from the PRROC package provided by Grau et al. (2015).

Interaction between DKK1 and DACT2 using $\theta \in\left\{\theta_{N}, \theta_{T}\right\}$ - Dual interactions $D A C T 2<>-<>D K K 1$ and $D K K 1 \mid-<>D A C T 2(D A C T 2 \mid-$ $\mid D K K 1$ and $D K K 1<>-\mid D A C T 2$ ) in normal (tumor) sample were found as depicted in figure 9 Figure 18 shows the kernel density estimate of the predicted conditional probabilities for both normal and tumor test cases. Using the weighted mean of the discretized values of the test samples (discretization done using median estimated from the training data as mentioned before), the predicted $\operatorname{Pr}(D K K 1 \mid D A C T 2)$ and $\operatorname{Pr}(D A C T 1 \mid D K K 1)$ are classified as active 
or passive. It might be useful to note that instead of using 0.5 as an arbitrary value, the weighted mean captures the distribution of labels in a much more realistic manner and helps infer interactions among the factors in the Wnt pathway.

Note the distributions depicted in figure 18. In the first column of the figure, the median for $\operatorname{Pr}(D K K 1 \mid D A C T 2)$ in normal (tumor) case is 0.4853088 (0.5006437). These medians point to the mid value of the belief in the genegene interaction depicted by the range of predicted conditional probabilities. The weighted threshold $\theta_{N}^{D K K 1}\left(\theta_{T}^{D K K 1}\right)$ based on labels for normal (tumor) test case was estimated at 0.5138889 (0.4861111). The estimations come from the following computations in equation 9 -

$$
\begin{aligned}
& \theta_{N}^{D K K 1}=\frac{1 \times n_{1, N}+2 \times n_{2, N}}{(1+2) \times\left(n_{1, N}+n_{2, N}\right)}=\frac{1 \times 264+2 \times 312}{3 \times 576}=0.5138889 \\
& \theta_{T}^{D K K 1}=\frac{1 \times n_{1, T}+2 \times n_{2, T}}{(1+2) \times\left(n_{1, T}+n_{2, T}\right)}=\frac{1 \times 312+2 \times 264}{3 \times 576}=0.4861111
\end{aligned}
$$

Similarly, in the second column of the figure, the median for $\operatorname{Pr}(D A C T 2 \mid D K K 1)$ in normal (tumor) case is $0.5606946(0.2985911)$. The weighted threshold $\theta_{N}^{D A C T 2}$ $\left(\theta_{T}^{D A C T 2}\right)$ based on labels for normal (tumor) test case was estimated at 0.4583333 (0.5416667). The estimations come from the following computations in equation 10 -

$$
\begin{aligned}
\theta_{N}^{D A C T 2} & =\frac{1 \times n_{1, N}+2 \times n_{2, N}}{(1+2) \times\left(n_{1, N}+n_{2, N}\right)}=\frac{1 \times 360+2 \times 216}{3 \times 576}=0.4583333 \\
\theta_{T}^{D A C T 2} & =\frac{1 \times n_{1, T}+2 \times n_{2, T}}{(1+2) \times\left(n_{1, T}+n_{2, T}\right)}=\frac{1 \times 216+2 \times 360}{3 \times 576}=0.5416667(10)
\end{aligned}
$$

It can be observed that the discretization is more realistic and strict using the weighted threshold rather than using the arbitrary value of 0.5 . The multiple peaks point to the different frequencies at which the predicted probabilities were recorded. Note that the probabilities here represent the belief in the activation status and the discretization only calibrates the belief into active and repressed state. To evaluate the results further wet lab tests are needed.

Using these distributions and distributions obtained using arbitrary value, the respective ROC are plotted and corresponding AUC values estimated. Finally, KS test is used to find the existence of statistical significance between the valid permutations of the distributions. These estimates further help derive insights about the interactions at a computational level. Figure 19 shows the ROC plots and the respective AUC values for the dual interactions observed via the in silico experiments. The following are compared -

1. labels of test data $g e_{N}$ and discretized values of $\operatorname{Pr}(D K K 1 \mid D A C T 2)$ and $\operatorname{Pr}(D A C T 2 \mid D K K 1)$ using weighted mean in Normal case

2. labels of test data $g e_{T}$ and discretized values of $\operatorname{Pr}(D K K 1 \mid D A C T 2)$ and $\operatorname{Pr}(D A C T 2 \mid D K K 1)$ using weighted mean in Tumor case 

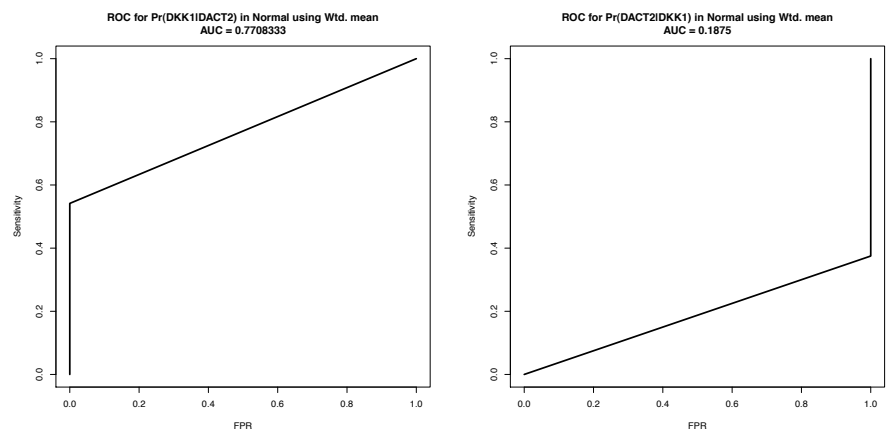

(a) $D A C T 2<>-<>D K K 1$ in Normal

(b) $D K K 1 \mid-<>D A C T 2$ in Nor-

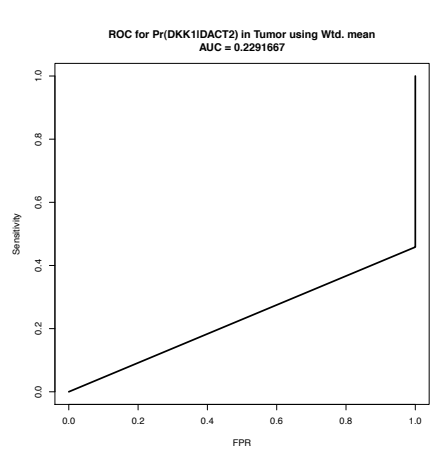

(c) $D A C T 2|-| D K K 1$ in Tumor mal

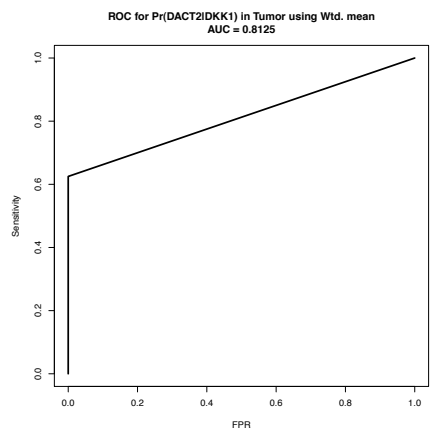

(d) $D K K 1<>-\mid D A C T 2$ in Tumor

Figure 19: Column wise ROCs for $\operatorname{Pr}(D K K 1 \mid D A C T 2)\left(1^{\text {st }}\right.$ column $)$ and $\operatorname{Pr}(D A C T 2 \mid D K K 1)$ $\left(2^{\text {nd }}\right.$ column) have been plotted with ETGN value for the $90 \%$. Row wise the plots depict the curves generated using weighted mean for Normal case and weighted mean for Tumor case. Respective AUC values for the ROC curves appear on the title of each of the graphs.

In figure 19 column wise the ROCs for $\operatorname{Pr}(D K K 1 \mid D A C T 2)\left(1^{\text {st }}\right.$ column $)$ and $\operatorname{Pr}(D A C T 2 \mid D K K 1)\left(2^{\text {nd }}\right.$ column) have been plotted with ETGN value for the $90 \%$. Row wise the plots depict the curves generated using weighted mean for Normal case and weighted mean for Tumor case. It can be seen that using the weighted mean, the subfigure $19(\mathrm{a})$ and $19(\mathrm{~d})$ convey a good guess regarding the type of interaction prevailing in normal and tumor case. Thus $D A C T 2<>-<>D K K 1$ i.e $\operatorname{Pr}(D K K 1 \mid D A C T 2)$ is highly favoured in Normal case while $D K K 1<>-\mid D A C T 2$ i.e $\operatorname{Pr}(D A C T 2 \mid D K K 1)$ is highly favoured in Tumor case. Why this is so is because the normal cases show better results in terms of prediction in comparison to the tumor cases. This points to the fact that the interaction $D A C T 2<>-<>D K K 1$ is strongly supported in the normal case in comparison to $D A C T 2|-| D K K 1$ which is weakly supported in the tumor case. Even though the algorithm showed that interaction 
was reversible at computational level, ROC curves and corresponding AUC values indicate weakness in the belief that $D A C T 2|-| D K K 1$ prevails in tumor cases. On the other hand, the interaction depicted by $\operatorname{Pr}(D A C T 2 \mid D K K 1)$ shows higher predictive quality in the tumor case with respect to the normal case. This means that $D K K 1<>-\mid D A C T 2$ has more weight in tumor case than its reversible $D K K 1 \mid-<>D A C T 2$ counter part in the normal case. Taken together, the dual interactions do exist but with different strengths of belief as shown conditional probability values. The curves in subfigure 19(b) and 19(c) indicate a bad guess and thus do not support the interactions $D K K 1 \mid-<>D A C T 2$ i.e $\operatorname{Pr}(D A C T 2 \mid D K K 1)$ in Normal case and $D A C T 2|-| D K K 1$ i.e $\operatorname{Pr}(D K K 1 \mid D A C T 2)$ in Tumor case.

Interaction between DKK1 and DACT2 using $\theta=0.5$

In comparison to use of the weighted $\theta$, the analysis of single interaction using $\theta=0.5$ is also presented. Figure 8 shows the interaction between $D K K 1$ and $D A C T 2$ as $D A C T 2<>-\mid D K K 1$, i.e $\operatorname{Pr}(D K K 1 \mid D A C T 2)$. Using a 0.5 threshold on 18 it is possible to see that discretization of kernel density estimates of $\operatorname{Pr}(D K K 1 \mid D A C T 2)$ induces a degree of belief which is not exactly $0(1)$. This is not the case with $\operatorname{Pr}(D A C T 2 \mid D K K 1)$, were the discretization leads to an exact 0(1) which removes the degree of belief. Bayesian networks often represent the degree of belief in terms of some real valued number and exact probabilities of $0(1)$ are considered with suspicion.

Figure 20 shows the ROC plots and the respective AUC values for the dual interactions observed via the in silico experiments. The following are compared

1. labels of test data $g e_{N}$ and discretized values of $\operatorname{Pr}(D K K 1 \mid D A C T 2)$ and $\operatorname{Pr}(D A C T 2 \mid D K K 1)$ using arbitrary value of 0.5 in Normal case

2. labels of test data $g e_{T}$ and discretized values of $\operatorname{Pr}(D K K 1 \mid D A C T 2)$ and $\operatorname{Pr}(D A C T 2 \mid D K K 1)$ using arbitrary value of 0.5 in Tumor case

In figure 20 column wise the ROCs for $\operatorname{Pr}(D K K 1 \mid D A C T 2)\left(1^{\text {st }}\right.$ column) and $\operatorname{Pr}(D A C T 2 \mid D K K 1)\left(2^{\text {nd }}\right.$ column) have been plotted with ETGN value for the $90 \%$. Row wise the plots depict the curves generated using arbitrary threshold of 0.5 for Normal case and Tumor case. It can be seen that using the a value of 0.5 , the subfigure 20 (a) conveys a negligibly good guess regarding the type of interaction prevailing in normal. Thus $D A C T 2<>-\mid D K K 1$ i.e $\operatorname{Pr}(D K K 1 \mid D A C T 2)$ is highly favoured in Normal case. On the other hand the 20(c) conveys a very bad guess regarding the reversal of interaction in Tumor case for $\operatorname{Pr}(D K K 1 \mid D A C T 2)$. Finally, it was noted that the degree of belief in $\operatorname{Pr}(D A C T 2 \mid D K K 1)$ was not at all recorded via thresholding. Thus even though 20(b) and 20(d) show recorded ROCs but the discretization of 0.5 does not capture the involved interaction. Thus the arbitrary value of 0.5 is not a good factor for inferring interactions.

Comparing figures 20 and 19, it is clear that the later gives a better guess in terms of the interpretation of the interaction obtained by discretizing the kernel density estimates of inferred conditional probabilities. To evaluate the statistical significance of the predicted probabilities, the values of the KS test 

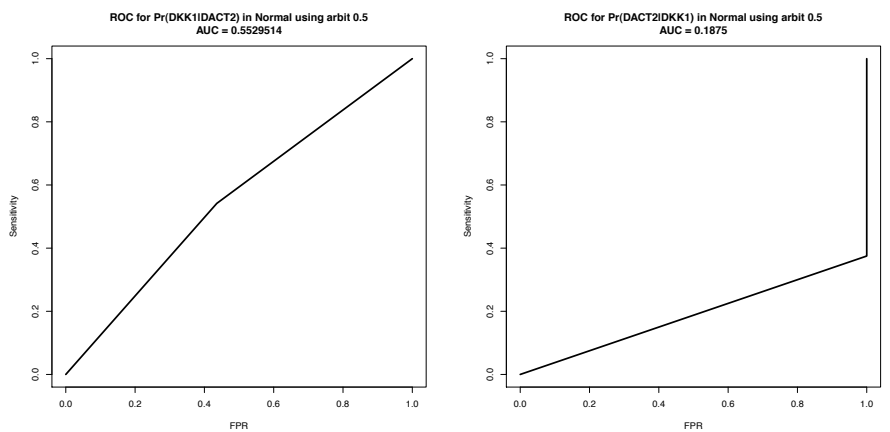

(a) $D A C T 2<>-\mid D K K 1$ in Normal

(b) $\operatorname{Pr}(D A C T 2 \mid D K K 1) \quad$ not
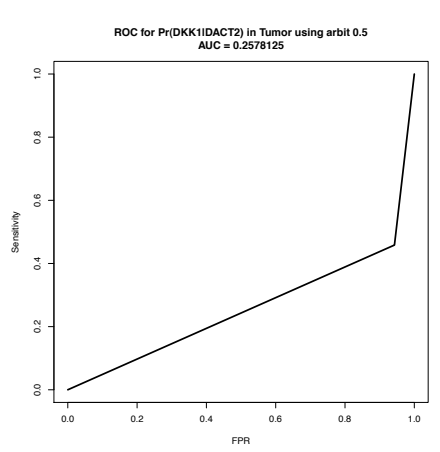

(c) $D A C T 2 \mid-<>D K K 1$ in Tu- recorded in Normal

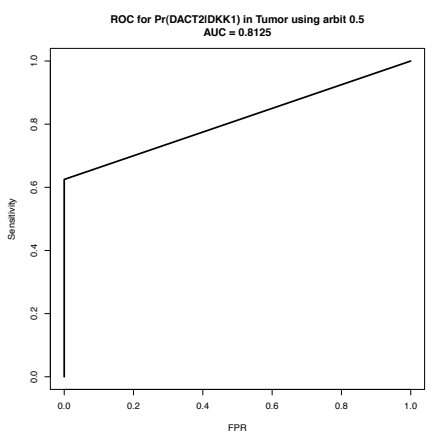

(d) $\operatorname{Pr}(D A C T 2 \mid D K K 1) \quad \operatorname{not}$

recorded in Tumor

Figure 20: Column wise ROCs for $\operatorname{Pr}(D K K 1 \mid D A C T 2)\left(1^{\text {st }}\right.$ column $)$ and $\operatorname{Pr}(D A C T 2 \mid D K K 1)$ ( $2^{\text {nd }}$ column) have been plotted with ETGN value for the $90 \%$. Row wise the plots depict the curves generated using arbit value of 0.5 for Normal case and arbit value of 0.5 for Tumor case. Respective AUC values for the ROC curves appear on the title of each of the graphs.

are tabulated and analyzed. Table 11 represents the computed values. The first four rows show the existing significance between the predictions for which the ROC curves have be plotted and described earlier. The next describes the significance between predictions based on thresholds for both normal and tumor cases. Note that some tests show no significance at all as is the case with $\operatorname{Pr}(D A C T 2 \mid D K K 1)$. In general, significance values differ depending on different interactions. Finally, significance values between interactions are also tabulated. It was found that there exists statistical difference between the inferred dual interactions as shown by the low p-values. Similar interpretations can be derived and respective measures can be plotted from the in silico observations. 


\begin{tabular}{|c|c|c|c|c|}
\hline \multicolumn{5}{|c|}{ Kolmogorov-Smirnov test } \\
\hline \multirow{2}{*}{ Sr. No. } & Discretized Val. vs Labels & p-value & Discretized Val. vs Labels & $\mathrm{p}$-value \\
\hline & \multicolumn{2}{|c|}{$\operatorname{Pr}(D K K 1 \mid D A C T 2)$} & \multicolumn{2}{|c|}{$\operatorname{Pr}(D A C T 2 \mid D K K 1)$} \\
\hline 1. & wtd. mean $(\mathrm{N})$ vs $g e_{N}$ & $\begin{array}{c}\mathrm{D}=0.5417 \\
\mathrm{p} \text {-value }<2.2 e^{-16}\end{array}$ & wtd. mean $(\mathrm{N})$ vs $g e_{N}$ & $\begin{array}{c}\mathrm{D}=0.625 \\
\mathrm{p} \text {-value }<2.2 e^{-16}\end{array}$ \\
\hline 2. & wtd. mean $(\mathrm{N})$ vs $g e_{N}$ & $\begin{array}{c}\mathrm{D}=0.1059 \\
\mathrm{p} \text {-value }=0.003129\end{array}$ & wtd. mean $(\mathrm{N})$ vs $g e_{N}$ & $\begin{array}{c}\mathrm{D}=0.625 \\
\text { p-value }<2.2 e^{-16}\end{array}$ \\
\hline 3. & wtd. mean $(\mathrm{N})$ vs $g e_{T}$ & {$\left[\begin{array}{c}\mathrm{D}=0.5417 \\
\text { p-value }<2.2 e^{-16}\end{array}\right.$} & wtd. mean $(\mathrm{T})$ vs $g e_{T}$ & $\begin{array}{c}\mathrm{D}=0.625 \\
\text { p-value }<2.2 e^{-16}\end{array}$ \\
\hline 4. & wtd. mean $(\mathrm{N})$ vs $g e_{T}$ & $\begin{array}{c}\mathrm{D}=0.4844 \\
\text { p-value }<2.2 e^{-16}\end{array}$ & wtd. mean $(\mathrm{T})$ vs $g e_{T}$ & $\begin{array}{c}\mathrm{D}=0.625 \\
\text { p-value }<2.2 e^{-16}\end{array}$ \\
\hline \multicolumn{5}{|c|}{ KS test between predictions using wtd. mean and arbitrary value of 0.5} \\
\hline \multirow{2}{*}{$\begin{array}{c}\text { Sr. No. } \\
1 .\end{array}$} & $\operatorname{Pr}(D K K 1 \mid D A C T 2)$ & KS value & $\operatorname{Pr}(D K K 2 \mid D A C T 1)$ & KS value \\
\hline & & $\begin{array}{c}\mathrm{D}=0.4358 \\
\text { p-value }<2.2 e^{-16}\end{array}$ & wtd. mean vs arbit. (N) & $\begin{array}{c}\mathrm{D}=0 \\
\mathrm{p} \text {-value }=1\end{array}$ \\
\hline 2. & wtd. mean vs arbit. (T) & $\begin{array}{c}\mathrm{D}=0.0573 \\
\mathrm{p} \text {-value }=0.3009\end{array}$ & wtd. mean vs arbit. (T) & $\begin{array}{c}\mathrm{D}=0 \\
\mathrm{p} \text {-value }=1\end{array}$ \\
\hline \multicolumn{5}{|c|}{ KS test between predictions of interactions $I_{1}$ and $I_{2}$} \\
\hline 2. & $\begin{array}{l}\text { wtd. mean }-I_{1}(\mathrm{~N}) \text { vs } I_{2}(\mathrm{~N}) \\
\text { arbit. }-I_{1}(\mathrm{~N}) \text { vs } I_{2}(\mathrm{~N})\end{array}$ & \begin{tabular}{|c|}
$\mathrm{D}=1$ \\
$\mathrm{p}$-value $<2.2 e^{-16}$ \\
$\mathrm{D}=0.5642$ \\
$\mathrm{p}$-value $<2.2 e^{-16}$
\end{tabular} & $\begin{array}{l}\text { wtd. mean - } I_{1}(\mathrm{~T}) \text { vs } I_{2}(\mathrm{~T}) \\
\text { arbit. - } I_{1}(\mathrm{~T}) \text { vs } I_{2}(\mathrm{~T})\end{array}$ & $\begin{array}{c}\mathrm{D}=1 \\
\mathrm{p} \text {-value }<2.2 e^{-16} \\
\mathrm{D}=0.9427 \\
\mathrm{p} \text {-value }<2.2 e^{-16}\end{array}$ \\
\hline
\end{tabular}

Table 11: Kolmogorov-Smirnov test indicating statistical significance between the distribution of predictions. Statistical significance is evaluated by observing the p-value. Small p-value indicates that significant difference. Significance test is conducted between (1) discretized values of predictions and existing test labels (2) discretized values of predictions based on weighted threshold and discretized values of predictions based on arbit threshold and (3) between predictions representing the dual interactions (obtained using both thresholds). $I_{1}$ and $I_{2}$ correspond to interactions inferred from $\operatorname{Pr}(D K K 1 \mid D A C T 2)$ and $\operatorname{Pr}(D A C T 2 \mid D K K 1)$, respectively.

\section{Caveats}

This work does not take into account the time series data which contains much more crucial information rather than the static data of gene expression. The inferences have been made regarding a natural phenomena based on the exploration of a computational causal model via sensitivity analysis. The results discussed are based on deviations of inferred conditional probabilities which encode a degree of belief in the occurrence of an event. Even if dynamic bayesian models are used, the observations will be made on degree of beliefs only. Also, the current bayesian network model does not encode the cyclic feedback loops. This has serious implications in the fact that the model might not capture correct interactions. The problem can be overcome to a certain extent by encoding the biological knowledge such that concepts of $d$-connectivity/separability exploit the inherent prior knowledge and thus help in proper inferences. More specifically, the model captures a snapshot in time but by varying the parameters or the prior/conditional probability tables, it is possible to verify the natural phenomena under investigation. 


\section{Future directions}

In context of the above observations, dynamic models might reveal greater information regarding the psychophysical laws. Work by Goentoro and Kirschner (2009) employs sensitivity analysis methods to reveal such laws by tuning single parameters. There might be a few ways to measure fold change in single an multi parameter settings. Future work might involve deeper study of the phenomena based on multi-parameter setting in a dynamic bayesian network model. If one incorporates nodes in between two time snapshots of $\beta$-catenin concentration in a dynamic bayesian network, one might be able to measure the changes at different phases of the signaling pathway. For example, in figure 21 a set of nodes measuring the different concentrations of $\beta$-catenin (say $N$ ) are depicted. In a dynamic bayesian network, the previous concentration at $t$ is connected to the next concentration at $t+1$. Also, to measure the effect of difference $(\Delta N)$, a change in concentration can be measured. Computations regarding fold change $(\Delta N)$ could then be estimated as posterior probabilities given the two concentrations, which the Bayesian networks can easily handle. In case more parameters need to be involved (say the effect of Wnt and APC together), nodes might be added as shown below. Then the fold change is conditional on $N(t+1), N(t+2), \Delta W n t$ and $\triangle A P C$ and is estimated as $\operatorname{Pr}(\Delta N(t+1) \mid N(t+1), N(t+2), \Delta W n t, \Delta A P C)$.

Regarding sensitivity analysis, in nonlinear problems, it might be useful to use Sobol' (1990) indices to estimate the sensitivity of the parameters. These indices are a way to estimate the changes in a multiparameter setting thus helping one to conduct global sensitivity analysis instead of local sensitivity analysis Glen and Isaacs (2012). Finally, with respect to the robustness of the gene-gene interaction network, the current work employs a very simple algorithm to construct the network and infer preserved interactions across the range of values set for a particular parameter. This helps in eliminating interactions that do not contribute enough biological information in the pathway or are non existant and require further analysis by integration of more data. Work in these lines would require incorporation of bigger datasets.

\section{Conclusions}

In this preliminary work via sensitivity analysis, the variation in predictive behaviour of $\beta$-catenin based transcription complex conditional on gene evidences is shown to follow power-logarithmic psychophysical law crudely. This implies deviations in output are proportional to increasing function of deviations in the input and show constancy for higher values of input. This points towards stability in the behaviour of transcriptional activity downstream of the Wnt pathway. As a further development, computational analysis shows that the preserved gene-gene interactions are also subject to these power-logarithmic psychophysical laws. The prevalence of these laws is reported for interaction between elements of pairs of (SFRP3, MYC), (SFRP2, CD44) and (DKK1, $D A C T 2)$. As a precursor to the analysis of these laws at interaction level, 


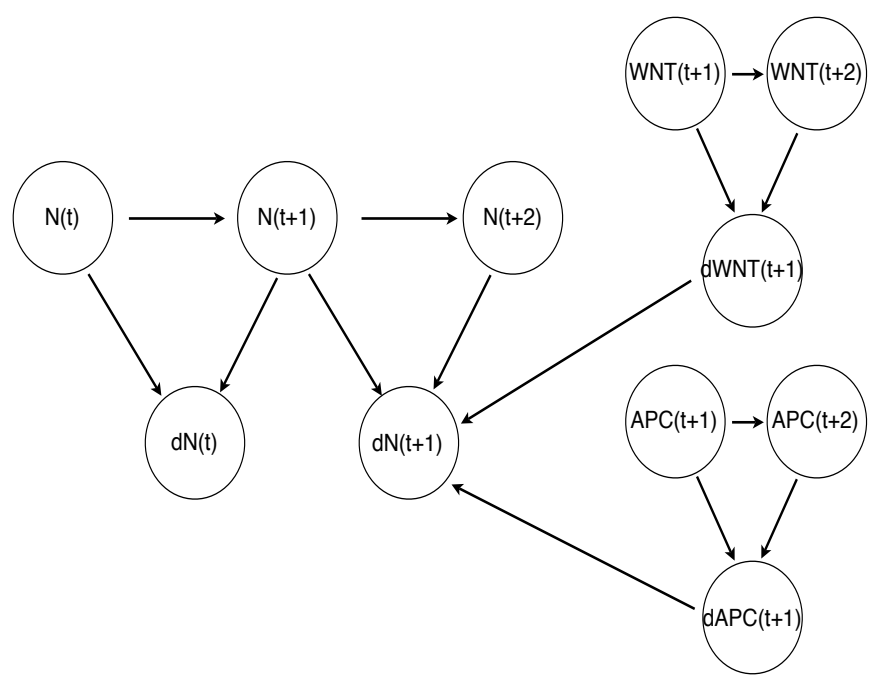

Figure 21: A schematic diagram of a dynamic bayesian network model that might help study the fold change and the logarithmic psychophysical laws behind the changes.

the biologically inspired epigenetically influenced computational causal models were used to infer gene-gene interaction from conditional probabilities of individual gene activation given the status of another gene activation. In relation of colorectal cancer cases, it is now possible to infer the type of interaction that might be happening among the genes at a pair wise level using BN models and further wet lab studies can be developed to investigate the inferred prevalence of power-logarithmic psychophysical laws at interaction level within the pathway. To assert the fact, in a recent development via wet lab experiments by Olsman and Goentoro (2016), it has been confirmed that there are existence of sensors that behave in a logarithmic fashion. The wet lab work by Olsman and Goentoro (2016) supports the earlier proposed crude postulates based on computational sensitivity analysis of this manuscript regarding the existence of logarithmic behaviour in the signaling pathways. It also signifies the importance of systems biology approach where in silico experiments combined with in vivo/in vitro experiments have the power to explore the deeper mechanisms of a signaling pathway.

\section{Acknowledgement}

Thanks to - • Dr. Uri Alon from Weizmann Institute of Science, Israel, for lively and critical discussion on behaviour of curves at the International Conference on Systems Biology of Human Disease (2015), held at German Cancer 
Research Center in Heidelberg, Germany. - Dr. Silja Renooij for providing useful feedback and editing the manuscript. - the Royal Society of Chemistry (RSC) for giving permission to reproduce parts of material in S. Sinha, Integr. Biol., 2014, DOI: 10.1039/C4IB00124A. • all anonymous reviewers who have helped in refining this manuscript.

\section{Declaration of Interest}

No conflict of interest.

\section{Appendix}

\subsection{Steps for construction of gene gene interaction networks}

Before starting the construction of interactions from the conditional probabilities, assign a variable $g g_{I}$ as an empty list (say in R language). Then $\forall i$ genes, execute the following -

1. $\forall 576$ runs iterated by a counter $j$

(a) append $x_{N}$ with the vector whose elements are $\operatorname{Pr}\left(g_{i}=\right.$ active $\mid g_{k}$ evidence) $\forall k$ genes in the $j^{t h}$ run for Normal test sample. This creates a matrix at the end of the runs.

(b) append $x_{T}$ with the vector whose elements are $\operatorname{Pr}\left(g_{i}=\right.$ active $\mid g_{k}$ evidence) $\forall k$ genes in the $j^{t h}$ run for Tumor test sample. This creates a matrix at the end of the runs.

(c) append $g e_{N}$ with the vector whose elements are $g e_{k}$ evidence $\forall k$ genes in the $j^{\text {th }}$ run for Normal test sample. This creates a matrix at the end of the runs.

(d) append $g e_{T}$ with the vector whose elements are $g e_{k}$ evidence $\forall k$ genes in the $j^{\text {th }}$ run for Tumor test sample. This creates a matrix at the end of the runs.

2. assign variables $g e, a a_{N}, a r_{N}, r a_{N}, r r_{N}, a a_{T}, a r_{T}, r a_{T}, r r_{T}, P g g N, P g g T$ to an empty vector $c()$ (say in R language). Note - a (r) means activation (repression).

3. compute mean across columns of $x N$ and $x T$ to obtain averaged $\widehat{\operatorname{Pr}}_{N}\left(g_{i} \mid g_{k}\right)$ and $\widehat{\operatorname{Pr}}_{T}\left(g_{i} \mid g_{k}\right) \forall k$ gene evidences and $\forall i$ genes. Note $k, i \in 1, \ldots, n$ if $n$ is the total number of genes.

4. assign a vector of $\widehat{\operatorname{Pr}}_{N}\left(g_{i} \mid g_{k}\right) \forall k$ genes to $P g g N$ and a vector of $\widehat{\operatorname{Pr}}_{T}\left(g_{i} \mid g_{k}\right)$ $\forall k$ genes to $P g g T$

5. $\forall k$ genes except the $i^{t h}$ one

(a) $\operatorname{if}(k \neq i)$

i. assign variables $\operatorname{tmpaa}_{N}$, tmpar $_{N}$, tmpra $_{N}, \operatorname{tmprr}_{N}$, tmpaa $_{T}$, tmpar $_{T}$, tmpra $_{T}$ and tmprr $_{T}$ to 0.

ii. assign threshold values $\theta$ to either a fixed value (say 0.5 ) or a weighted mean. 
iii. if assigning a weighted mean, compute the threshold $\theta_{N}$ as the weighted mean of the labels of the test data i.e evidences for the $i^{t h}$ gene, in the case of Normal samples (top formula in equation 8). Similarly, compute the threshold $\theta_{T}$ as the weighted mean of the labels of the test data i.e evidences for the $i^{\text {th }}$ gene, in the case of Tumor samples (bottom formula in equation 8).

iv. $\forall 576$ runs iterated by a counter $l$

A. if $\left(g e_{N}[l, k]==1\right.$ and $\left.x_{N}[l, k]<\theta\right)$ increment tmprr $_{N}$ by 1

B. else if $\left(g e_{N}[l, k]==1\right.$ and $\left.x_{N}[l, k]>=\theta\right)$ increment $\operatorname{tmpar}_{N}$ by 1

C. else if $\left(g e_{N}[l, k]==2\right.$ and $\left.x_{N}[l, k]<\theta\right)$ increment tmpra $_{N}$ by 1

D. else if $\left(g e_{N}[l, k]==2\right.$ and $\left.x_{N}[l, k]>=\theta\right)$ increment tmpaa $a_{N}$ by 1

E. if $\left(g e_{T}[l, k]==1\right.$ and $\left.x_{T}[l, k]<\theta\right)$ increment $\operatorname{tmprr}_{T}$ by 1

F. else $\operatorname{if}\left(g e_{T}[l, k]==1\right.$ and $\left.x_{T}[l, k]>=\theta\right)$ increment $\operatorname{tmpar}_{T}$ by 1

G. else if $\left(g e_{T}[l, k]==2\right.$ and $\left.x_{T}[l, k]<\theta\right)$ increment tmpra $_{T}$ by 1

H. else if $\left(g e_{T}[l, k]==2\right.$ and $\left.x_{T}[l, k]>=\theta\right)$ increment tmpaa ${ }_{T}$ by 1

v. Comment - store results

vi. append ge with $g_{k}, r r_{N}$ with $t m p r r_{N}, a r_{N}$ with $\operatorname{tmpar}_{N}, r a_{N}$ with tmpra $_{N}, a a_{N}$ with $t m p a a_{N}, r r_{T}$ with $\operatorname{tmprr}_{T}, a r_{T}$ with tmpar $_{T}, r a_{T}$ with tmpra $_{T}$ and $a a_{T}$ with tmpaa $_{T}$

(b) store the variables in the previous step to a data frame (say in $\mathrm{R}$ language) to a variable stats.

(c) Comment - 1 means aa, 2 means ar, 3 means ra, 4 means rr

(d) assign variables $g g_{I N}$ and $g g_{I T}$ as empty vector []

(e) $\forall j$ gene except the $i^{\text {th }}$ one under consideration

i. find the index $i d x_{N}$ in stats that corresponds to 1 or 2 or 3 or 4

ii. if $\left(i d x_{N}==1\right)$ append $g g_{I N}$ with interaction string stats $\S g_{j}<>$ $-<>g_{i}$

iii. else if $\left(i d x_{N}==2\right)$ append $g g_{I N}$ with interaction string stats§ge ${ }_{j} \mid-<>$ $g_{i}$

iv. else if $\left(i d x_{N}==3\right)$ append $g g_{I N}$ with interaction string $s t a t s \S g_{j}<>$ $-\mid g_{i}$

v. else if $\left(i d x_{N}==4\right)$ append $g g_{I N}$ with interaction string $\operatorname{stats} \S g_{j} \mid-$ $\mid g_{i}$

vi. find the index $i d x_{N}$ in stats that corresponds to 1 or 2 or 3 or 4 vii. if $\left(i d x_{T}==1\right)$ append $g g_{I T}$ with interaction string $s t a t s \S g_{j}<>$ $-<>g_{i}$

viii. else if $\left(i d x_{T}==2\right)$ append $g g_{I T}$ with interaction string stats§g $g_{j} \mid-<>$ $g_{i}$

ix. else if $\left(i d x_{T}==3\right)$ append $g g_{I T}$ with interaction string $\operatorname{stats} g_{j}<>$ $-\mid g_{i}$ 
x. else if $\left(i d x_{T}==4\right)$ append $g g_{I T}$ with interaction string $\operatorname{stats} \oint g_{j} \mid-$

$\mid g_{i}$

(f) assign stats§gg $g_{I N}$ with $g g_{I N}$

(g) assign stats $\S g g_{I T}$ with $g g_{I T}$

(h) Comment $-i^{\text {th }}$ gene influenced

(i) $g g_{I}[[i]]<-\operatorname{list}\left(i g=g_{i}\right.$, stats $=$ stats, PggN $=\operatorname{PggN}$, PggT $=$ $\operatorname{PggT}$ )

\section{References}

Adler, M., Mayo, A., Alon, U., 2014. Logarithmic and power law input-output relations in sensory systems with fold-change detection. PLoS computational biology 10, e1003781.

Baehs, S., Herbst, A., Thieme, S., Perschl, C., Behrens, A., Scheel, S., Jung, A., Brabletz, T., Göke, B., Blum, H., et al., 2009. Dickkopf-4 is frequently down-regulated and inhibits growth of colorectal cancer cells. Cancer letters 276, 152-159.

Baron, R., Kneissel, M., 2013. Wnt signaling in bone homeostasis and disease: from human mutations to treatments. Nature medicine 19, 179-192.

Bayes, M., Price, M., 1763. An essay towards solving a problem in the doctrine of chances. Philosophical Transactions 53, 370 .

Bernoulli, D., 1738. Specimen theoriae novae de mensura sortis. Commentarii Academiae Scientiarum Imperialis Petropolitanae 5, 175-192.

Blagodatski, A., Poteryaev, D., Katanaev, V., 2014. Targeting the wnt pathways for therapies. Mol Cell Ther 2, 28.

Brent, R., Lok, L., 2005. A fishing buddy for hypothesis generators. Science 308, 504.

Brophy, P., Lang, K., Dressler, G., 2003. The secreted frizzled related protein 2 (sfrp2) gene is a target of the pax2 transcription factor. Journal of Biological Chemistry 278, 52401-52405.

Caldwell, G., Jones, C., Taniere, P., Warrack, R., Soon, Y., Matthews, G., Morton, D., 2006. The wnt antagonist sfrp1 is downregulated in premalignant large bowel adenomas. British journal of cancer 94, 922-927.

Charniak, E., 1991. Bayesian networks without tears. AI magazine 12, 50.

Clevers, H., 2006. Wnt/[ $\beta]$-catenin signaling in development and disease. Cell 127, 469-480.

Costello, J., Plass, C., 2001. Methylation matters. Journal of medical genetics 38, 285-303.

Curtin, J.C., Lorenzi, M.V., 2010. Drug discovery approaches to target wnt signaling in cancer stem cells. Oncotarget 1, 552 .

Das, P., Singal, R., 2004. Dna methylation and cancer. Journal of Clinical Oncology 22, 4632-4642.

Fechner, G.T., 1860. Elemente der Psychophysik (2 vols). Breitkopf and Hartel.

Feng Han, Q., Zhao, W., Bentel, J., Shearwood, A., Zeps, N., Joseph, D., Iacopetta, B., Dharmarajan, A., 2006. Expression of sfrp- 4 and $\beta$-catenin in human colorectal carcinoma. Cancer letters $231,129-137$.

Friedman, N., Linial, M., Nachman, I., Pe'er, D., 2000. Using bayesian networks to analyze expression data. Journal of computational biology 7, 601-620. 
Garber, K., 2009. Drugging the wnt pathway: problems and progress. Journal of the National Cancer Institute 101, 548-550.

Gardner, T., Faith, J., 2005. Reverse-engineering transcription control networks. Physics of Life Reviews 2, 65-88.

Glen, G., Isaacs, K., 2012. Estimating sobol sensitivity indices using correlations. Environmental Modelling \& Software 37, 157-166.

Goentoro, L., Kirschner, M.W., 2009. Evidence that fold-change, and not absolute level, of $\beta$-catenin dictates wnt signaling. Molecular Cell 36, 872-884.

González-Sancho, J., Aguilera, O., García, J., Pendás-Franco, N., Peña, C., Cal, S., de Herreros, A., Bonilla, F., Muñoz, A., 2004. The wnt antagonist dickkopf-1 gene is a downstream target of $\beta$-catenin/tcf and is downregulated in human colon cancer. Oncogene 24, 1098-1103.

Grau, J., Grosse, I., Keilwagen, J., 2015. Prroc: computing and visualizing precision-recall and receiver operating characteristic curves in $\mathrm{r}$. Bioinformatics, btv153.

Gregorieff, A., Clevers, H., 2005. Wnt signaling in the intestinal epithelium: from endoderm to cancer. Genes \& development 19, 877-890.

Hartemink, A., Gifford, D., Jaakkola, T., Young, R., 2001. Using graphical models and genomic expression data to statistically validate models of genetic regulatory networks, in: Pac. Symp. Biocomput, Citeseer. pp. 422-433.

Hoang, B., Moos, M., Vukicevic, S., Luyten, F., 1996. Primary structure and tissue distribution of frzb, a novel protein related to drosophila frizzled, suggest a role in skeletal morphogenesis. Journal of Biological Chemistry 271, 26131-26137.

Huang, D., Yu, B., Deng, Y., Sheng, W., Peng, Z., Qin, W., Du, X., 2010. Sfrp4 was overexpressed in colorectal carcinoma. Journal of cancer research and clinical oncology 136, 395-401.

Issa, J., 2007. Dna methylation as a therapeutic target in cancer. Clinical Cancer Research 13, 1634-1637.

Jiang, X., Tan, J., Li, J., Kivimäe, S., Yang, X., Zhuang, L., Lee, P., Chan, M., Stanton, L., Liu, E., Cheyette, B., Yu, Q., 2008. Dact3 is an epigenetic regulator of wnt/ $\beta$-catenin signaling in colorectal cancer and is a therapeutic target of histone modifications. Cancer cell 13, 529-541.

Kahn, M., 2014. Can we safely target the wnt pathway? Nature Reviews Drug Discovery 13, $513-532$.

Kanwar, S., Yu, Y., Nautiyal, J., Patel, B., Majumdar, A., 2010. The wnt/ $\beta$-catenin pathway regulates growth and maintenance of colonospheres. Molecular cancer 9, 212.

Kivimäe, S., Yang, X., Cheyette, B., 2011. All dact (dapper/frodo) scaffold proteins dimerize and exhibit conserved interactions with vangl, dvl, and serine/threonine kinases. BMC biochemistry 12,33 .

Kriegl, L., Horst, D., Reiche, J., Engel, J., Kirchner, T., Jung, A., et al., 2010. Lef-1 and tcf4 expression correlate inversely with survival in colorectal cancer. J. Transl. Med 8, 123.

Masin, S.C., Zudini, V., Antonelli, M., 2009. Early alternative derivations of fechner's law. Journal of History of the Behavioral Sciences 45, 56-65. doi 10.1002/jhbs.20349

Matsui, A., Yamaguchi, T., Maekawa, S., Miyazaki, C., Takano, S., Uetake, T., Inoue, T., Otaka, M., Otsuka, H., Sato, T., et al., 2009. Dickkopf-4 and-2 genes are upregulated in human colorectal cancer. Cancer science 100, 1923-1930.

Murphy, K., et al., 2001. The bayes net toolbox for matlab. Computing science and statistics 33, 1024-1034. 
Needham, C.J., Bradford, J.R., Bulpitt, A.J., Westhead, D.R., 2007. A primer on learning in bayesian networks for computational biology. PLoS computational biology 3, e129.

Niehrs, C., 2006. Function and biological roles of the dickkopf family of wnt modulators. Oncogene $25,7469-7481$.

Olsman, N., Goentoro, L., 2016. Allosteric proteins as logarithmic sensors. Proceedings of the National Academy of Sciences, 201601791.

Pearl, J., 1988. Probabilistic reasoning in intelligent systems: networks of plausible inference. Morgan Kaufmann.

Pearl, J., 2000. Causality: models, reasoning, and inference. Cambridge Univ Pr.

Pećina-S̆laus, N., 2010. Wnt signal transduction pathway and apoptosis: a review. Cancer Cell International 10, 1-5.

Pendas-Franco, N., Garcia, J., Pena, C., Valle, N., Palmer, H., Heinäniemi, M., Carlberg, C., Jimenez, B., Bonilla, F., Munoz, A., et al., 2008. Dickkopf-4 is induced by tcf/ $\beta$-catenin and upregulated in human colon cancer, promotes tumour cell invasion and angiogenesis and is repressed by $1 \alpha, 25$-dihydroxyvitamin d3. Oncogene $27,4467-4477$.

Peterson, C., Laniel, M., et al., 2004. Histones and histone modifications. Current Biology 14, 546-551.

Peer, D., Regev, A., Elidan, G., Friedman, N., 2001. Inferring subnetworks from perturbed expression profiles. Bioinformatics 17, S215.

Pinto, D., Gregorieff, A., Begthel, H., Clevers, H., 2003. Canonical wnt signals are essential for homeostasis of the intestinal epithelium. Genes \& development 17, 1709-1713.

Rao, T.P., Kühl, M., 2010. An updated overview on wnt signaling pathways a prelude for more. Circulation research 106, 1798-1806.

Reguart, N., He, B., Xu, Z., You, L., Lee, A., Mazieres, J., Mikami, I., Batra, S., Rosell, R., McCormick, F., et al., 2004. Cloning and characterization of the promoter of human wnt inhibitory factor-1. Biochemical and biophysical research communications 323, 229-234.

Roehrig, S., 1996. Probabilistic inference and path analysis. Decision Support Systems 16, 55-66.

Sachs, K., Gifford, D., Jaakkola, T., Sorger, P., Lauffenburger, D., 2002. Bayesian network approach to cell signaling pathway modeling. Sci. STKE $148,38-42$.

Sachs, K., Perez, O., Pe'er, D., Lauffenburger, D., Nolan, G., 2005. Causal protein-signaling networks derived from multiparameter single-cell data. Science 308, 523.

Sato, H., Suzuki, H., Toyota, M., Nojima, M., Maruyama, R., Sasaki, S., Takagi, H., Sogabe, Y., Sasaki, Y., Idogawa, M., Sonoda, T., Mori, M., Imai, K., Tokino, T., Shinomura, Y., 2007. Frequent epigenetic inactivation of dickkopf family genes in human gastrointestinal tumors. Carcinogenesis 28, 2459-2466.

Schmidt-Ott, K., Masckauchan, T., Chen, X., Hirsh, B., Sarkar, A., Yang, J., Paragas, N., Wallace, V., Dufort, D., Pavlidis, P., et al., 2007. $\beta$-catenin/tcf/lef controls a differentiation-associated transcriptional program in renal epithelial progenitors. Development 134, 3177-3190.

Shannon, P., Markiel, A., Ozier, O., Baliga, N., Wang, J., Ramage, D., Amin, N., Schwikowski, B., Ideker, T., 2003. Cytoscape: a software environment for integrated models of biomolecular interaction networks. Genome research 13, 2498-2504.

Sharma, R., 1973. Wingless a new mutant in drosophila melanogaster. Drosophila information service $50,134-134$.

Sheather, S.J., Jones, M.C., 1991. A reliable data-based bandwidth selection method for kernel density estimation. Journal of the Royal Statistical Society. Series B (Methodological) , 683-690. 
Sinha, S., 2014. Integration of prior biological knowledge and epigenetic information enhances I] the prediction accuracy of the bayesian wnt pathway. Integr. Biol. 6, 1034-1048. doi 10.1039/ c4ib00124a

Sobol', I.M., 1990. On sensitivity estimation for nonlinear mathematical models. Matematicheskoe Modelirovanie 2, 112-118.

Sokol, S., 2011. Wnt Signaling in Embryonic Development. volume 17. Elsevier.

Strahl, B., Allis, C., 2000. The language of covalent histone modifications. Nature 403, 41-45.

Sun, J.Z., Wang, G.I., Goyal, V.K., Varshney, L.R., 2012. A framework for bayesian optimality of psychophysical laws. Journal of Mathematical Psychology 56, 495-501.

Suzuki, H., Watkins, D., Jair, K., Schuebel, K., Markowitz, S., Chen, W., Pretlow, T., Yang, B., Akiyama, Y., Van Engeland, M., et al., 2004. Epigenetic inactivation of sfrp genes allows constitutive wnt signaling in colorectal cancer. Nature genetics 36, 417-422.

Taniguchi, H., Yamamoto, H., Hirata, T., Miyamoto, N., Oki, M., Nosho, K., Adachi, Y., Endo, T., Imai, K., Shinomura, Y., 2005. Frequent epigenetic inactivation of wnt inhibitory factor-1 in human gastrointestinal cancers. Oncogene 24, 7946-7952.

Thorstensen, L., Lind, G.E., Løvig, T., Diep, C.B., Meling, G.I., Rognum, T.O., Lothe, R.A., 2005. Genetic and epigenetic changes of components affecting the wnt pathway in colorectal carcinomas stratified by microsatellite instability. Neoplasia 7, 99-108.

Veeck, J., Dahl, E., 2012. Targeting the wnt pathway in cancer: the emerging role of dickkopf-3. Biochimica et Biophysica Acta (BBA)-Reviews on Cancer , 18-28.

Voronkov, A., Krauss, S., 2012. Wnt/beta-catenin signaling and small molecule inhibitors. Current pharmaceutical design 19, 634 .

Waterman, M., 2004. Lymphoid enhancer factor/t cell factor expression in colorectal cancer. Cancer and Metastasis Reviews 23, 41-52.

Weber, E.H., 1834. De pulsu resorptione, auditu et tactu. Annotationes anatomicae et physiologicae.

Yochum, G., 2011. Multiple wnt/ $\beta$-catenin responsive enhancers align with the myc promoter through long-range chromatin loops. PloS one 6, e18966.

Yu, J., Virshup, D.M., 2014. Updating the wnt pathways. Bioscience reports 34, 593-607.

Yuan, G., Wang, C., Ma, C., Chen, N., Tian, Q., Zhang, T., Fu, W., 2012. Oncogenic function of dact1 in colon cancer through the regulation of $\beta$-catenin. PloS one 7 , e34004.

Zhong, Z., Ethen, N.J., Williams, B.O., 2014. Wnt signaling in bone development and homeostasis. Wiley Interdisciplinary Reviews: Developmental Biology 3, 489-500.

Zitt, M., Untergasser, G., Amberger, A., Moser, P., Stadlmann, S., Zitt, M., Müller, H., Mühlmann, G., Perathoner, A., Margreiter, R., et al., 2008. Dickkopf-3 as a new potential marker for neoangiogenesis in colorectal cancer: expression in cancer tissue and adjacent non-cancerous tissue. Disease Markers 24, 101-109. 